\title{
Collisions d'ions lourds à haute énergie, aspects théoriques (*)
}

\section{J. Cugnon}

Institut de Physique B5, Université de Liège, Sart Tilman,

B 4000 Liège 1, Belgique

Résumé : La dynamique des collisions entre ions lourds dans la région 250-2000 MeV/R est étudife dans la perspective de la formation de matière nucléaire dense et chaude et de l'extraction possible de propriétés statiques de cette matière, notamment de l'énergie de compression. Aprés un rappel concernant l'équation d'etat, on étudie la dynamique de la collision dans le cadre de l'hydrodynamique et de la cascade intranucléaire. Le contexte théorique de l'équation de LandauVlassov est largement étudié et permet une comparaison entre les deux modảes, une étude de la thermalisation possible et de l'expansion du système. Enfin, certaines informations sur I'équation d'état sont extraites en analysant la multiplicité de pions, la production de fragments et l'écoulement collectif. A divers endraits, les extensions vers les basses énerqies sont discutées.

Abstract: The dynamics of the heavy ion collisions in the 250-2000 MeV/A energy range is studied in relation with the formation of hot, dense nuclear matter and with the possibility of extracting the compressional energy. After a short reminder of the properties of the equation of state, the collision dynamics is studied both in the hydrodynamical and the intranuclear cascade models. The LandauVlassov equation is largely studied, which permits a comparison between the two madels, an investigation of the possible thermalisation and of the expansion of the system. Finally, information about the equation of state is extracted from the pion multiplicity, the composite yield and the collective flow. On many occasions, extension toward lower energy is discussed.

\section{INTRODUCTION}

Le but avoué ou non de l'étude des collisions entre ions lourds à haute énergie est de déterminer l'équation d'état de la matière nucléaire. En effet, à cause de l'énergie disponible et en admettant une opacité nucléaire raisonnable, le processus de collision conduit inévitablement à la formation d'un système dense et fortement excité ${ }^{1}$. Cette perspéctive a soulevé au début un grand enthousiasme, car, à part certaines indications astrophysiques, c'etait la première fois que I'on avait un moyen d'étudier l'équation d'état. Cependant, très vite, cet enthousiasme a fait place à une certaine deception. On était do plus en plus persuadé que l'expansion et la désagrégation du système, qui suivent la période de compression, effaçaient en quelque sorte les proprietés de la matière dense. De plus, il était évident que le système ne pouvait etre en équilibre, ne serait-ce que pendant la période initiale de compression. A l'heure actuelle, l'optimisme 
est à nouveau répandu, grâce à divers développements importants pendant les cinq ou six dernières années.

Un premier progrès a d'abord été obtenu grăce à la confection d'un modele, le modele de cascade intranucléaire ${ }^{2-4}$, qui peut prendre en compte des effets de nonéquilibre de la maniere la plus satisfaisante possible.

L'enthousiasme reprit lorsque, avec la référence 5 , on a découvert que certaines grandeurs "résistent" à l'expansion, c'est-à-dire conservent leur valeur pendant la phase d'expansion. Ces grandeurs contiennent done des informations sur l'équation d'état.

Mais il a faliu attendre une démarche originale de R. Stock ${ }^{6}$ qui, en dépit de l'existence d'effets hors équilibre, proposa un moyen d'extraire des informations, limitées il est vrai, sur les proprietés de la matière dense. L'idée est de comparer d'une façon adéquate les résultats expérimentaux et les résultats de la cascade intranucléaire. Cette méthode a été appliquée a la multiplicité de pions, la production de composites et l'écoulement collectif.

Ce qui suit est l'exposé de ces développements, où, en plus, on insiste sur les notions d'équation d'état, d'équilibre lacal, d'effets hors équilibre et de validité des concepts thermodynamiques. A beaucoup d'occasions, nous discutons aussi ces notions dans le contexte des basses énergies. La Section 2 traite de l'équation d'état. La Section 3 est dévolue à la discussion de la dynamique de la réaction. Dans la Section 4, on aborde le probleme de la thermalisation et de l'équilibre local. On aborde les propriétés présumées de la matière dense dans la Section 5, ainsi que la notion de grandeurs résistant à l'expansion. Enfin, la

Section 6 décrit comment on tente d'extraire l'équation d'état.

\section{EQUATION D'ETAT}

\subsection{Rappels de thermodynamique}

Pour un système thermadynamique, dont le nombre de particules n'est pas fixé, les propriétés sant contenues dans le potentiel thermodynamique (ou grand potentiel)

$$
\Omega=\Omega(u, V, T),
$$

où $\mu$ est le potentiel chimique, $V$ le volume et 1 la température. Le grand potentiel vaut

$$
\Omega=-p V,
$$

ce qui donne automatiquement la pression. Les variables thermodynamiques exten sives (l'entropie et le nombre moyen de particules) sont obtenues par dérivation

$$
S=-\frac{\partial \Omega}{\partial T} \quad, \quad A=-\frac{\partial \Omega}{\partial \mu} .
$$

Les autres grandeurs extensives s'obtiennent alars grâce aux définitions usuelles. 
L'énergie interne U yaut

et l'energie libre

$$
F=\Omega+\mu A \quad .
$$

Pour un système infini, le volume $V$ perd son statut de variable thermodynamique indépendante et les variables extensives perdent leur sens. On considère plutôt les densités en volume. Dés lors, les équations $(2.1)-(2.2)$ se réduisent à

$$
p=p(\mu, T)
$$

et on peut écrire $\left(s=\lim _{V \rightarrow \infty} \frac{S}{V}, \rho=\lim _{V \rightarrow \infty} \frac{A}{V}\right)$

$$
s=-\left(\frac{\partial p}{\partial T}\right)_{\mu}, \quad p=-\left(\frac{\partial p}{\partial \mu}\right)_{T},
$$

et

$$
\begin{aligned}
& u=p+T s+u p, \\
& f=p+\mu \rho,
\end{aligned}
$$

En physique nucléaire, on a pris I'habitude de parler des quantités par nucléon (ce qui revient à considérer les densités massiques). On pourra écrire

$$
\begin{aligned}
& \frac{\Omega}{A}=-\frac{P}{\rho}, \\
& \frac{S}{A}=-\frac{1}{\rho}\left(\frac{\partial P}{\partial T}\right)_{H}, \quad 1=-\frac{1}{\rho}\left(\frac{\partial P}{\partial H}\right)_{T}, \\
& \frac{U}{A}=-\frac{P}{\rho}+T \frac{S}{A}+\mu,
\end{aligned}
$$

Cette dernière relation montre qu'a l'équilibre a $T$ et $\mu$ donnés $(p=0)$, on a $\mu=\frac{F}{A}$. On a aussi

$$
\frac{S}{A}=-\frac{\partial}{\partial T}\left(\frac{F}{A}\right)_{\rho}, \quad p=\rho^{2} \frac{\partial}{\partial \rho}\left(\frac{F}{A}\right)_{T}, p=\rho^{2} \frac{\partial}{\partial \rho}\left(\frac{U}{A}\right)_{S} / A .
$$

\subsection{Equation d'etat}

D'une manière générale, I'équation d'état est une relation entre trois variables thermodynamiques intensives. Cela peut être $p=p(\mu, T)$, ou $p=p(p, T)$ ou $u=u(p, p)$. Une forme commode et usuelle est 


$$
\frac{U}{A}=\frac{U}{A}(\rho, r) .
$$

En toute généralité, on peut écrire

$$
\frac{U}{A}(\rho, T)=\frac{U}{A}(\rho, 0)+\left[\frac{U}{A}(\rho, T)-\frac{U}{A}(\rho, 0)\right]
$$

ou

$$
\frac{U}{A}(\varphi, T)=E_{c}(D)+E_{t h}(\varphi, T)
$$

Les quantite apparaissant dans (2.15) sont habituellement appelées énergie de compression et énergie thermique, respectivement. Elles ne doivent pas être confondues avec le travail et la quantité de chaleur échangés pendant n'importe quelle transformation, ces derniers ne pouvant être des fonctions d'état.

Une autre manière de présenter l'équation détat est de considérer les isothermes

$$
p=p(p, T)
$$

c'est-à-dire les courbes donnant la pression pour une température fixée, au les isoentropes

$$
p=p\left(p, \frac{S}{A}\right)
$$

ou encore

$$
p=p(u, T) .
$$

Toutes ces relations ne sont pas équivalentes. Celles donnant une variabie en fonction de ses variables indépendantes naturelles contiennent l'information totale : $p=p(u, T), \frac{U}{A}=\frac{U}{A}\left(\frac{S}{A}, T\right), \frac{F}{A}=\frac{F}{A}(\rho, T)$. Pour s'en convaincre, il suffit de rappeler que pour un gas parfait non relativiste, $(2.16)$ s'écrit toujours $p=p r$ $(k=1)$, alors que $(2.17)$ s'écrira $p=2 u v^{-1}$ où $v$ est le nombre de degrés de liberté mis en jeu.

\subsection{Rappels de mécanique statistique}

La quantité cruciale est le calcul de la fonction de partition du systeme

$$
Z=\operatorname{Tr} \exp [-B(H-\mu N)]
$$

où $B=\frac{1}{T}$ est l'inverse de la température et $\mu$ le potentiel chimique. $H$ est l'hamil tonien du système et $N$ est 1 'opérateur nombre de particules. $Z$ est relié au grand potentiel par 
De $(2.3),(2.4)$ et $(2.18)$ on tire, par exemple

$$
U=\operatorname{Ir}\{H \exp [-B(H-\mu N)]\}=\langle H\rangle,
$$

où les crochets indiquent la même moyenne que celle impliquée par la trace. Le calcul exact de (2.18) est généralement impossible pour des systemes de particules en interaction. On en est done réduit à utiliser des approximations.

Une autre manière de décrire un système à l'équilibre est de regarder la fonction densité $\rho\left(\overrightarrow{\vec{r}}, \overrightarrow{\vec{z}^{\prime}}\right)$

$$
\rho\left(\vec{r}, \vec{r}^{\prime}\right)=\left\langle\psi^{+}(\vec{r}) \psi\left(\vec{r}^{\prime}\right)\right\rangle
$$

ou aussi sa transformée de Wigner

$$
f_{1}(\vec{r}, \vec{p})=\int \frac{d^{3} S}{(2 \pi)} e^{i \vec{p} \cdot \vec{s}} \rho\left(\vec{r}+\frac{\vec{s}}{2}, \vec{r}-\frac{\vec{s}}{2}\right) .
$$

Cette fonction intégrée sur $\vec{p}$ donne la densité en $\vec{r}$, et intégrée sur $\vec{I}$ donne la densité dans l'espace des impulsions. Elle se comporte donc comme la fonction de distribution à un corps classique, qui donne la probabilité de trouver une particule avec l'impulsion $\vec{p}$ à la position $\vec{r}$. Dans un systeme etendu avec des interractions à courte portée, la densité à l'équilibre est uniforme. D'autres fonctions sont aussi intéressantes. Ainsi, la fonction de distribution à deux corps

$$
f_{2}\left(\vec{r}_{1}, \vec{p}_{1}, \vec{r}_{2}, \vec{p}_{2}\right)=\int \frac{d^{3} S_{1}}{(2 \pi)^{3}} \frac{d^{3} S_{2}}{(2 \pi)^{3}}\left\langle\psi^{+}\left(\vec{r}_{1}+\frac{\vec{S}_{1}}{2}\right) \psi^{+}\left(\vec{r}_{2}+\frac{\vec{S}_{2}}{2}\right) \psi\left(\vec{r}_{1}-\frac{\vec{s}_{2}}{2}\right) \psi\left(\vec{r}_{2}-\frac{\vec{s}_{2}}{2}\right)\right\rangle
$$

dont 1 'interpretation est la probabilite de trouver une particule en $\left(\vec{r}_{1}, \vec{p}_{1}\right)$ et en meme temps une particule en $\left(\vec{I}_{2}, \vec{p}_{2}\right)$. Une grandeur qui en dérive est la fonction da corrélation (classique)

$$
v(\vec{r})=\int d^{3} p_{1} d^{3} p_{2}\left[f_{2}\left(\vec{r}_{1}, \vec{p}_{1}, \vec{r}_{2}, \vec{p}_{2}\right)-f_{1}\left(\vec{r}_{1}, \vec{p}_{1}\right) f_{1}\left(\vec{r}_{2}, \vec{p}_{2}\right)\right]
$$

où nous avons anticipé sur le fait que pour un système infini, il y a invariance par translation et par rotation. la fonction $V(r)$ représente $l^{\text {itinfluence de la }}$ présence d'une particule sur la probabilité d'en trouver une autre à une distance r de la première.

Une autre quantité physique importante est la fonction de Green

$$
G^{+}\left(\vec{r}, \vec{r}^{\prime}, t^{\prime}, t^{\prime}\right)=\left\langle\psi^{+}(\vec{r}, t) \psi\left(\vec{r}^{\prime}, t^{\prime}\right)\right\rangle
$$

où le produit doit être ordonné dans le temps. Il est plus utile de considerer sa transformée de fourier

$$
G^{+}(k, \omega)=\int \frac{d^{3} s}{(2 \pi)^{3}} e^{i \vec{k} \cdot \vec{s}} \int d t e^{i \omega t} G^{t}(\vec{r}, \vec{r}-\vec{s}, t, t-\vec{t}),
$$

où, encore une fois, nous avons fait usage des propriétés d'invariance drun systeme étendu. On a bien sûr 


$$
D=\int \frac{d^{3} k}{(2 \pi)^{3}} \int \frac{d \omega}{2 \pi} C^{+}(k, \omega)
$$

La fonction de Green exprime les proprités â un corps du systeme. Diune manière générale, elle s'écrit

$$
G^{+}(k, \omega)=\frac{1}{\omega-\frac{k^{2} k^{2}}{2 m}-\Sigma(k, \omega)},
$$

ou 2 est la self-energie. Si lion appelle e(k) la valeur de w qui ennule le dénominateur pour un $k$ donné, on pourra écrire

$$
G^{+}(k, \omega) \approx \frac{\left.1+\frac{\partial \Sigma}{\partial \omega}\right)_{\omega=\varepsilon(k)}}{\omega-e(k)}
$$

Nous allons revenir bientat sur l'interpretation de cette formule.

A titre d'exemple, nous donnons rapidement la forme des grandeurs considérées pour un gaz de Boltzmann et pour un gaz de fermions. Nous encourageons les étudiants à écrire la même chose pour un gaz de bosons. L'hamiltanien est

$$
H=\sum_{k} e(k) a_{k}^{*} a_{k}
$$

et l'opérateur $N$ s'écrit

$$
N=\sum_{k} a_{k}^{+} a_{k}
$$

La fonction de partition s'écrit

$$
Z=\prod_{k}[\exp [-B(\operatorname{e}(k)-\mu)]+1]
$$

si ce sont des fermions,

$$
Z=\exp \left[\sum_{k} \exp [-B(e(k)-u)]\right\}
$$

si ce sont des Boltzmannions et

$$
z=\pi_{k}[1-\exp (-\beta(e(k)-\mu))]
$$

si ce sont des bosons. La pression s'écrit

$$
p=\frac{T}{V} \sum_{k} \ln \{1+\exp [B(\mu-e(k))]\}
$$

Le passage à la limite infinie (Iimite thermodynamique) se fait en remplaçant $v^{-1} \sum_{k}+g \frac{d^{3} k}{(2 \pi)^{3}}$, où g est la dégénérescence des états $\vec{k}$. D'ou : 


$$
p=g T \int \frac{d^{3} k}{(2 \pi)^{3}} \ln \{1+\exp [B(\mu-e(k))]\}
$$

pour les fermions et

$$
p=g T \int \frac{d^{3} k}{(2 \pi)^{3}} \exp [-\beta(e(k)-\mu)]
$$

pour les Boltzmannions. La densité (moyenne) de particules est

$$
\rho=\frac{A}{V} \equiv \int \frac{d^{3} k}{(2 \pi)^{3}} \frac{Q}{1+\exp [B(e(k)-u)]}
$$

pour les fermions et

$$
\rho=g \int \frac{d^{3} k}{(2 \pi)^{3}} e^{-\beta(e(k)-\mu))}
$$

pour les particules de Boltzmann. La densité d'énergie est

$$
u=g \int \frac{d^{3} k}{(2 \pi)^{3}} \frac{e(k)}{1+\exp [B(e(k)-\mu)]},
$$

$$
u=g \int \frac{d^{3} k}{(2 \pi)^{3}} e(k) \exp [-B(e(k)-\mu)],
$$

respectivement. La relation $u=\frac{3}{2} p$ n'est valable que $s i e(k)=\frac{k^{2} k^{2}}{2 m}$.

La densité d'entropie peut s'écrize (*)

$$
s=-g \int \frac{d^{3} k}{(2 \pi)^{3}}\{n(k) \ln n(k)-(1-n(k)) \ln (1-n(k))\}
$$

pour les fermions. Pour les Boltzmannions, le second terme tambe.

La fonction de distribution à un corps s'écrit bien sor

$$
f_{1}(\vec{r}, \vec{p})=g \exp (-\beta(E(k)-\mu))
$$

pour les Boltzmannions. La fonction de Green s'écrit

$$
G^{+}(\vec{k}, \omega)=\frac{1}{\omega-E(k)} .
$$

La fonction de corrélation $v(r)$ vaut 0 pour un gaz de Boltzmannions. Elle est différente de zéro pour les fermions parce que la statistique ne leur permet pas

d'etre au meme endroit. Pour $T=0$, elle est donné par

$$
1+\nu(r)=g(r)=-\delta_{\sigma \sigma^{1}} \frac{1}{8 \pi^{4}}\left(\frac{\sin k_{F} r}{r^{3}}-\frac{k_{F} \cos k_{F}{ }^{r}}{r^{2}}\right)^{2},
$$

oú $k_{F}$ est le moment de Fermi. Elle tend vers -1 quand $I+0$

Revenons a l'équation (2.30). Si la fonction de Green a seu près cette forme, cela veut dire que les particules en interaction se comportent à peu près comme des particules libres (quasi-particules) avec une énergie $e(k)$. Une grandeur 
importante est la masse effective $m^{*}$ definie par

$$
m *=k^{2}\left(\frac{\partial^{2} e(k)}{\partial k^{2}}\right)^{-1},
$$

qui représente l'inertie que cette quasi-particule a effectivement.

\subsection{Connaissances sur l'équation d'état}

La connaissance expérimentale de l'équation d'état se résume à peu de chase. En fait, on sait juste avec certitude que la fonction $E_{c}(\rho)$ a un minimum pour $\rho=p_{0} \approx$ $0.156 \pm 0.015 \mathrm{fm}^{-3}$, qui vaut $B=16 \pm 1 \mathrm{MeV}$. On sait aussi que le module de compressibilité

$$
K=q \frac{d}{d \rho}\left(\rho^{2} \frac{d E_{c}}{d \rho}\right)_{p=\rho_{0}}
$$

vaut $220 \mathrm{MeV} \pm 20 \mathrm{MeV}$

Du point de vue théorique, la situation est particulièrement décevante, ainsi qu'en témoigne la Figure 2.1, eu égard a l'énorme effort consenti dans les trente dernières années. Le calcul qui a été poussé le plus loin est dú à friedman et Pandharipande et est indiqué en trait plein sur la fiqure 2.1.

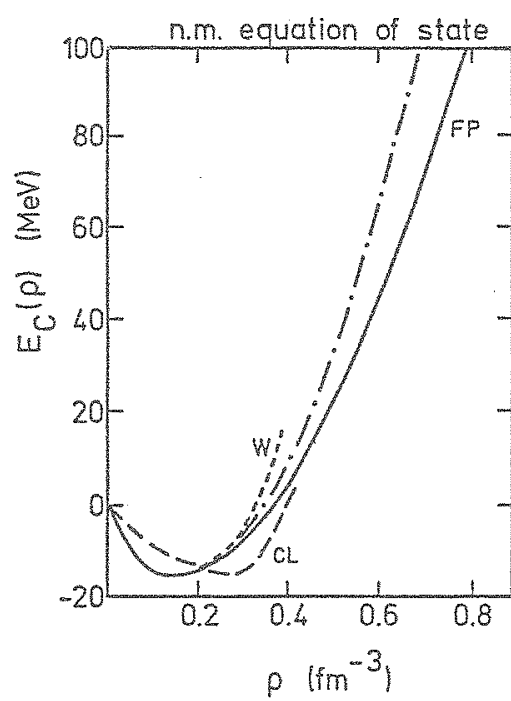

\section{Figure 2.1}

Prédictions théoriques pour l'énergie de compression. La courbe en trait plein est tirée d'un calcul variationel ${ }^{7}$. La courbe en traits discontinus est un calcul perturbatif à la Brueckner ${ }^{8}$ et la courbe pointillée est un calcul basé sur un modèle de champ moyen relativiste ${ }^{9}$. La courbe en trait/ point représente l'équation (2.48) avec les vâleurs expérimentales des paramètres.

Il contient cependant un parametre. C'est un calcul variationnel basé sur un hamiltonien de forces à deux corps réaliste et de forces à trois corps. La courbe marqué $\mathrm{CL}^{8}$ est basée sur un calcul perturbatif utilisant des forces à deux et à troi: corps sans aucun paramètre. La courbe en trait pointillé est basée sur un modèle de théorie des champs, du à Walecka". Deux parametres sont fixés pour obtenir la bonne saturation, mais il reste deux parametres libres.

La diversité des résultats theoriques est une source même de l'intérét portê 
a l'équation d'etat. En effet, Ia courbe marquée w dens la tigure 2.1 provient de

I'un des nouveaux modèles du noyau atomique. La connaissance expérimentale de l'équation d'etat permettrait de choisir parmi ces images physiques.

En plus de $E_{c}$, il serait interessant de connatre les isothermes sous la forme $p=p(p, T)$. Les théories existantes s'accordent ici sur un point : près de $p_{0}$, les isothermes sont du type de Vanderwals, c'est-a-dire sont celles d'une phase de co existence gaz-Iiquide. Nous renvoyons a ce sujet a l'exposé de P. Bonche ${ }^{10}$. Il serait aussi intéressant de connâtre les isoentropes $p=p\left(\rho, \frac{S}{A}\right)$, ou encore sous la forme $p=p\left(u, \frac{S}{A}\right)$. Dans la Figure 2.2 , nous mantrons les isothermes sous la forme $p=p(u, T)$ pour la matière neutronique, soulignant par la même occasion l'intérêt astrophysique de l'équation d'état en général.

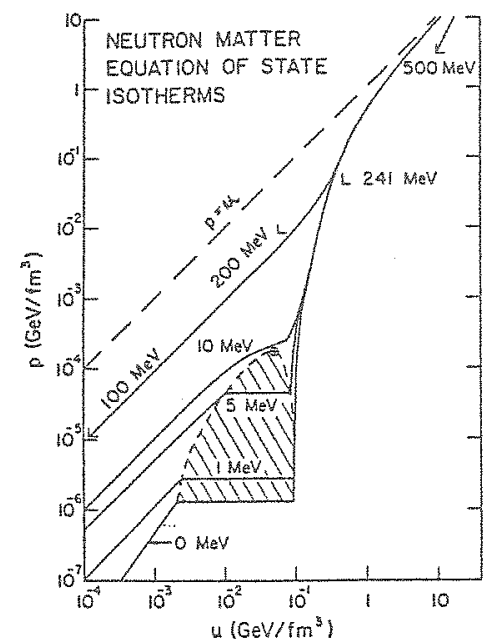

\section{Figure 2.2}

Fonctions $P=p(U, T)$ pour la matierre de neutrons, calculées suivant un modèle de champ moyen relativiste 9

Une équation d'état est dite dure si pour une énergie donnée et une température donnée la pression est grande. A ce sujet, il n'y a pas de point de référence bien spécifié. Souvent, on prend la forme la plus simple qui satisfait a l'observation, c'est-à-dire (pour $T=0$ )

$$
E_{c}=B+\frac{K}{18} \frac{\left(\rho-\rho_{0}\right)^{2}}{\rho_{0}^{2}} .
$$

Puisquielies doivent toutes passer par le point de saturation, on peut considerer la dureté d'une équation d'état par la dérivée seconde

$$
K^{\prime}=\frac{\partial}{\partial \rho}\left[\rho^{2} \frac{\partial}{\partial \rho}\left(\frac{U}{A}\right)\right]
$$

qui est reliée à la vitesse du sone。

$$
c_{0}=\sqrt{\frac{1}{M} \frac{\partial p}{\partial \rho}}=\sqrt{\frac{K T}{M}},
$$

a condition que toutes les dérivées partielles soient prises à $\frac{S}{A}$ constant. 
Dans le cas relativiste, cette relation devient $(c=1)$

$$
c_{0}=\sqrt{\frac{\partial p}{\partial u}}
$$

et montre que la dureté est liée à la croissance de $p$ avec u. En particulier, on voit que l'on ne peut avoir $p \geq u$ (si l'on a $p=0$ pour une valeur de $u$ ). Dans un diagramme tel que la Figure 2.2, la dureté est liée grosso-modo à la position de l'isotherme. On peut ayoir différentes équations d'état ayant des fonctions $K$ ' différentes pour un même paramètre $k$.

On peut voir facilement que l'équation (2.49) est inappropriée a haute densité. En effet, $s i p \gg p$, on peut voir que (2.12) peut s'ecrire $p \approx 2(u-B p)$, ce qui conduit à $c_{0}>1$. Ceci illustre qu'il est illusoire de décrire l'équation d'état à quelques $\rho_{0}$ à partir d'une équation telie que (2.49) (ou des semblables) qui ramenent la variation de la courbe à un seul parametre, $K$ en l'occurrence.

Dans la figure 2.3, nous montrons, a titre illustratif, certaines prédictions pour la masse effective. Les courbes résultent d'un modèle de champ moyen relativiste ${ }^{9}$, tandis que les croix résultent du calcul de la référence 8 . Dans ce dernier cas, la masse effective varie avec l'énergie. La valeur indiquée correspond à l'energie du niveau de fermi.

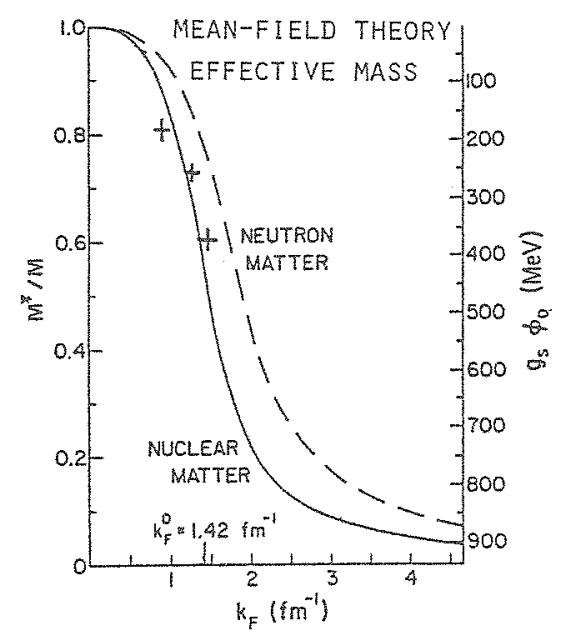

\section{Figure 2.3}

Prédictions théoriques de la masse effective. Voir texte.

\section{DYNAMIQUE DES COLLISIONS}

\subsection{Introduction}

Il est de plus en plus clair que la collision entre deux ions lourds dans la région du GeV par nucléon est un phénomène violent et rapide. Les résultats expérimentaux (voir la revue de R. Babinet ${ }^{11}$ ) abondent en ce sens. Nous rappellerons 
ici les plus significatifs :

1) Il y a une séparation plus ou moins nette entre participants et spectateurs, ces derniers étant peu perturbés.

2) Les participants sont émis a tous les angles, mais non isotropiquement, dans le centre de masse avec des spectres pius ou moins exponentiels. Les "températures" extraites sont reprises dans la figure 3.1 .

3) Des pions sont émis abondamment. Leur "temperature" est plus basse que celle des protons.

4) La zone participante émet également des particules composites: $d, t,{ }^{3} H e, \alpha, \ldots$

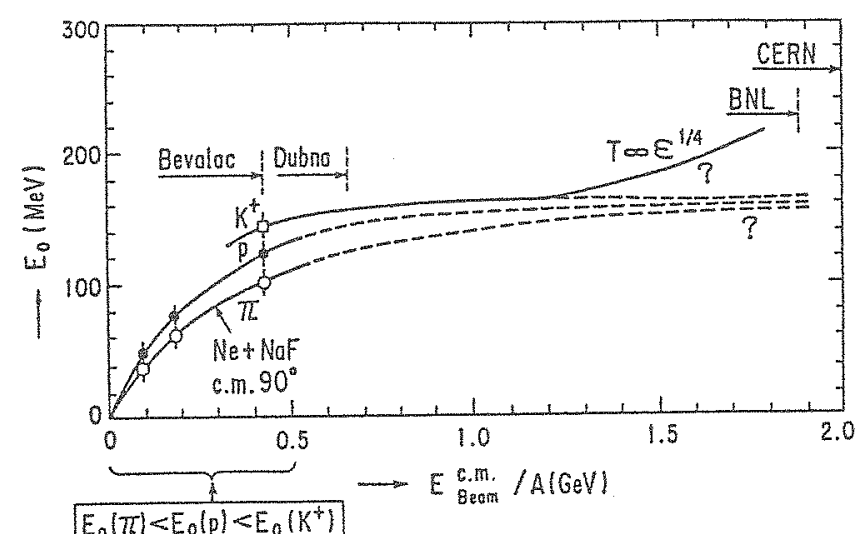

Figure 3.1. "Températures" extraites des queues des spectres de production à $90^{\circ} \mathrm{c} . \mathrm{m} .$, présentées en fonction de l'énergie par nucléon dans le c.m. Tiré de la Référence 12.

L'émission très abondante de particules de la zone participante et les hautes "temperatures" abservées indiquent, s'il le fallait, que la collision est un processus extrêmement violent, siège de phenomenes transients. Il semble dẻs lors hasardeux de vouloir extraire de l'observation de tels phénomènes des renseignements sur l'équation d'état, une propriété d'équilibre de la matière.

Afin d'étudier ces phénomènes, nous avons besoin d'une théorie dynamique (à l'opposé de statique) pour le problème à plusieurs corps. Dans la figure 3.2, nous avons repris un certain nombre de telles théories. De gauche a droite, on progresse du plus fondamental vers le plus "ordinaire". Du haut vers le bas, la description du systeme se simplifie, généralement par élimination des degrés de liberté, restreignant ainsi la complexité de la description du systeme (1'information). Il est inutile de dire que beaucoup de ces theories ne sont pas pratiques dans le sens que l'on ne peut générer des solutions, même au prix d'approximations numériques. De plus, il n'est pas nécessairement requis de choisir la théorie la plus fondamentale. En effet, le phenomène que l'on étudie réunit peutetre les conditions nécessaires pour qu'une des "réductions" de la théorie fondamentale soit suffisante. De plus, ainsi que nous en donnerons un exemple, la réduction thẹorique adéquate peut dépendre de l'observable que l'on veut étudier. 


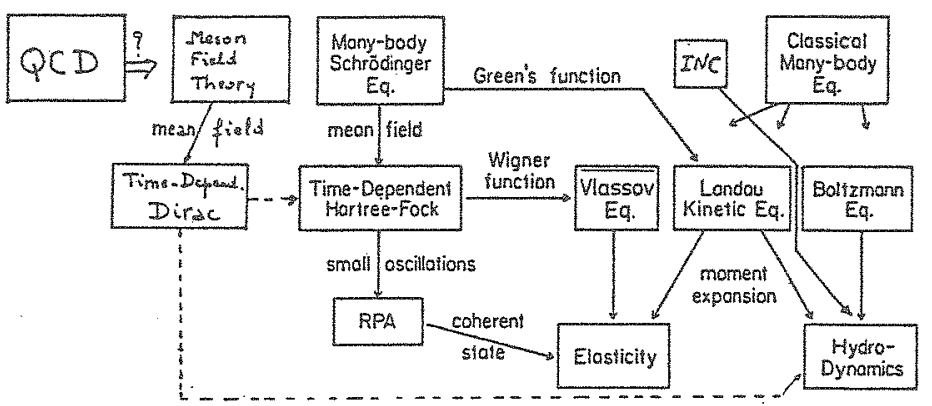

Figure 3.2. Comparaison de diverses théories dynamiques du problème à $N$ corps. Adapté de la Référence 13.

Essayons de dégager les conditions régnant pendant les collisions dans la région $250 \mathrm{MeV}$ - $2000 \mathrm{MeV} / \mathrm{A}$. Le mouvement relatif des deux noyaux peut etre traité classiquement. Dès lors, les sommes sur les parametres d'impact sont parfaitement justifiées. Déjà, le mouvement relatif de deux nucléons ne peut pas se traiter entierement classiquement, car même à $1 \mathrm{GeV} / \mathrm{c}$, le moment angulaire maximum est seulement $\ell_{\max } \approx 3$. Le point le plus délicat est le problème des interférences possibles entre les collisions nucléon-nucléon. Pour que celles-ci soient négligeables, il faudrait (au moins) que la portée des forces nucléaires $r_{s}$ soit petite vis-à-vis du libre parcours moyen $\lambda=\left(\rho \sigma_{N N}\right)^{-1}$, une condition qui n'est remplie que marginalement. En plus, il faut que le moment de transfert typique $\vec{q}$ soit plus grand que l'énergie de liaison d'un nucléon, ce qui aussi n'est pas très nettement vérifié. Ces deux conditions montrent que l'hypothèse de collisions nucléon-nucléon successives et indépendantes n'est pas amplement justifié et qu'à tout le moins les interactions à plusieurs corps sont présentes. La façon la plus simple d'en tenir compte est d'introduire également un champ moyen. De ceci, il résulte que l'équation de Landau-ylassov semble un bon point de départ pour décrire les collisions, encore qu'il serait souhaitable d'avoir une théorie qui traite un peu mieux le point de vue quantique du mouvement des nucléons, surtout dans la zone des énergies intermédiaires. Elle se révèle trop simpliste pour certaines observables, ainsi que nous le verrons. Néanmoins, cette approche jovera un rôle essentiel dans notre analyse.

Le domaine des collisions relativistes est caractérisé par deux aspects particuliers : les vitesses relativistes et la production de particules, de pions notamment. Le premier impose bien Évidemment la cinématique relativiste. Les aspects dynamiques relativistes (avec l'intraduction de spineurs) n'ont pas encore été étudiés dans le cadre des ions lourds. Nous nous limiterons, ainsi que généralement, à une production de pions prenant place lors de collisions entre baryons. Il est très difficile de voir quels paramètres demanderaient une description plus détallée, telle qu'une théorie utilisant des champs classiques de pions couplés a une densité de baryons, ce qui n'a pas encore tété vraiment fait. A un degré supérieur, on devrait tenir compte des fluctuations du champ, dans le cadre drune thérie quantique des champs. 
3.2. Hydrodynamique

\section{2 .1 . Eguations de base}

Dens cette approche ${ }^{14-16}$, on suppose que les noyaux se comportent comme des systemes fluides. Les équations hydradynamiques donnent la variation de grandeurs locales, macroscopiques, c'est-à-dire qui ne font pas référence aux degrés de liberté microscopiques. De plus, on supposera qu'il y a équilibre thermodynamique local, c'est-à-dire que l'état du système est donné par les grandeurs thermodynamiques hebituelles. Nous reviendrons sur tous ces aspects plus tard.

Les équations de base de I'hydrodynamique sont l'equation de continuité

$$
\frac{\partial \rho}{\partial t}+\vec{\nabla} \cdot(\rho \vec{u})=0
$$

où p est la densité de masse et $\vec{u}$ le champ de vitesse, et l'équation de Navierstokes ( $\eta, \tau$ sont les coefficients de viscositê usuels)

$$
\rho\left[\frac{\partial \vec{u}}{\partial t}+\vec{u} \cdot \vec{\nabla} \cdot \vec{u}\right]=-\vec{\nabla} p+\left[n\left(\Delta \vec{u}+\frac{1}{3} \vec{\nabla} \vec{\nabla} \cdot \vec{u}\right)+\zeta \vec{\nabla} \vec{\nabla} \cdot u\right]
$$

Cette équation n'exprime rien d'autre que la lai de Newton appliqué à un élément du fluide et traduit le transfert d'impulsion des degrés de liberté macroscopiques (u) vers des degrés de liberté internes. Le transfert d'énergie est décrit par

l'équation pour le champ de température (pour $\eta=\zeta=0$ )

$$
\left(\frac{\partial}{\partial t}+\vec{u} \cdot \vec{\nabla}\right) T=-\frac{T}{\rho C_{V}}\left(\frac{\partial p}{\partial T}\right)_{\rho} \vec{\nabla} \cdot \vec{u}+\frac{u}{\rho C_{V}} \Delta T,
$$

où $u$ est le coefficient de conductibilite thermique et $C_{V}$ est la chaleur spécifique a volume constant. Le système d'équations devient complet (six équations pou six inconnues, $\rho, \vec{u}, T, P$ ) si l'on y adjoint l'équation d'état

$$
p=p(\rho, T) \quad .
$$

Ceci rend cette approche particulièrement intéressante, puisqu'elle inclut l'équation d'état explicitement.

\subsubsection{Aspects numériques}

Résoudre les équations (3.1)-(3.4) dans leur totale complexité à trois dimensions $n^{3}$ pu être réalisé à ce jour. Ce qui peut être fait, et a été fait, est de résoudre sans terme de viscosite. On utilise généralement une technique de différences finies. Une conséquence curieuse de cette méthode est l'apparition d'effets de viscosité dus au procédé numerique, et done sans véritable controle. Cette viscosite semble assez faible cependant.

Expérimentalement, on ne mesure pas des quantités macroscopiques, mais plutot des particules individuelles. A un moment ou à un eutre, il faut done ré- 
introduire les degrés de liberté individuels. Ceci se fait en général en introduisant la notion de freeze-out. On admet que quand un élément de fluide atteint une densité de freeze-out $\rho_{f o}$ (utilisée comme un paramètre libre), cet élément est "gele" en un certain nombre moyen de particules, qui peuvent étre de différentes espaces $(n, p, \pi, \ldots)$. L'abondance relative de ces particules est supposée découler d'un équilibre chimique. Si l'on considère une seule espèce, la fonction de distribution à un corps devient done

$$
f_{1}\left(\vec{r}, \vec{p}, t_{f_{0}}\right)=\rho_{f_{0}}(\vec{r})(2 \pi m T(\vec{r}))^{-\frac{3}{2}} \exp \left[-\frac{(\vec{p}-m u(\vec{r}))^{2}}{2 m T(\vec{r})}\right] .
$$

La densité de freeze-out est choisie généralement entre $\rho_{0} / 3$ et $2 \rho_{0} / 3$.

\subsubsection{Dynamique de Ia collision}

La Figure 3.3 montre l'évolution de la densité du système Ar + Ca à $400 \mathrm{MeV}$ par nucléon. Pour $b=0$, on peut distinguer une phase de compression, qui dure jusqu'a $\sim 14 \mathrm{fm} / \mathrm{c}$, suivie d'une phase d'expansion. La caractéristique importante ici est l'émission de matière a $90^{\circ} \mathrm{com}$. Pour $b=4 \mathrm{fm}$, la région centrale est aussi le siège d'une compression importante. Cette matière se décomprime en poussant les "spectateurs" sur le coté, étant entendu que le terme "spectateurs" est plutôt mal utilisé ici, car en hydrodynamique toute la matière participe au processus. Pour les grands paramètres d'impact, la région centrale est très zéduite et n'apparact pas dans la distribution finale du courant (de masse).

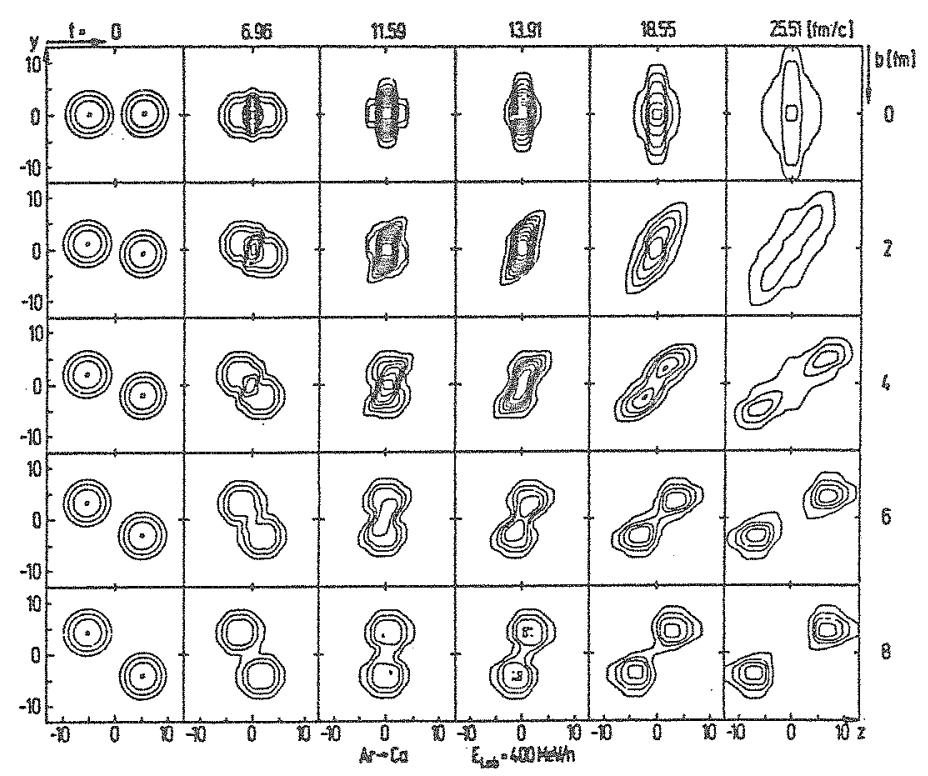


En premiere approche, on peut dire que la collision conduit à un état où la matière est dense et chaude, qui se désintegre en emettant beaucoup de particules.

On peut se demander si les propriétés de l'état final dépendent de l'équation d'état, ou plus important encore, si les propriétés de l'état final dépendent de l'équation d'état. La seconde question a été étudiée dans la Référence 18 . Ainsi que le montre la figure 3.4, les résultats sont très décevants. Dans la Référence 15, il est indiqué que le nombre de pions pourrait dépendre de l'équation d'état d'une façon notable. Nous reviendrons sur la première question dans la Section 5.

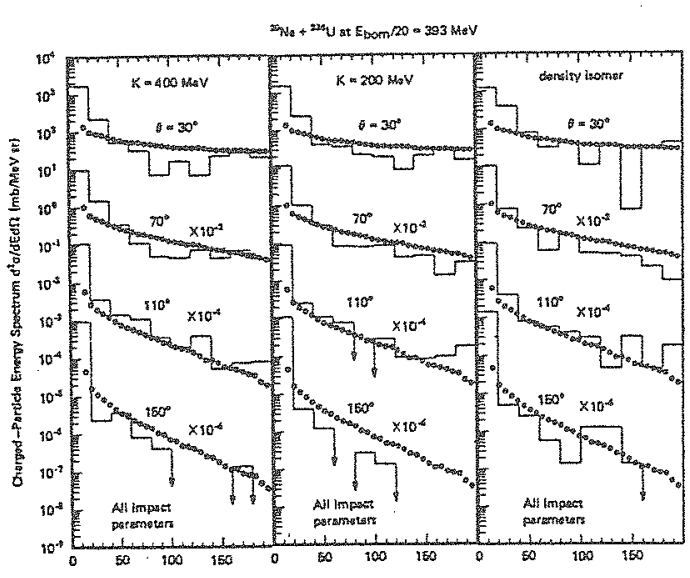

\section{Figure 3.4}

Comparaison entre la section efficace de production de charge ${ }^{17}$ et les calculs hydrodynamiques de la Référence 18. Trois équations d'état sont étudiées.

\subsection{Cascade intranucleaire}

\subsubsection{Description}

La cascade intranucléaire (C.I.) est basé sur une simulation des collisions Elle présente l'avantage (entre autres) de pouvoir traiter des problemes avec un grand nombre de degrés de liberté (jusqu'à des milliers) à un niveau micrascopique 19 .

La C.I décrit le processus de collision comme une suite de collisions binaires entre des particules sur couche et se produisant comme dans I'espace libre. En bref, les nucléons sont pourvus au début de positions et d'impulsionsen accord avec la distribution de masse des noyaux, le mouvement de fermi et l'énergie incidente du faisceau. Les particules se propagent en ligne droite, jusqu'à ce que pour une paire de particules la distance minimum d'approche $d_{\text {min }}$ soit réalisée. si celle-ci est trop grande, le mouvement n'est pas perturbé. Si, par contre,

$$
\pi d_{m i n}^{2} \leq \sigma_{t o t}\left(E_{c m}\right)
$$

où $\sigma_{\text {tot }}$ est la section efficace totale pour l'énergie $E_{\mathrm{cm}}$ de la paire en question, alors les particules seront déviées. Les impulsions des particules après la 
collision sont en accord, bien sur, avec les lois de conservation d'énergieimpuision, choisies pour simuler le distribution angulaire de collision. Si plusieurs voies sont ouvertes, la vaie rinale sera choisie en accord avec le poids des differentes sections efficaces de réction. Le mouvement rectilineaire des particules reprend jusqu'à ce qu'une nouvelle paire de particules réalise sa distance minimum d'approche, et ainsi de suite jusqu'à ce que le taux de collisions binaires devienne negligeable. Le ealcul est renouvelé plusieurs fois et les observables sont calculées par moyenne d'ensemble. D'autres aspects sont souvent introduits : la praduction de pions, la cinematique reletiviste, le principe de Pauil,...

Dans la C.I., on suit toutes les particules dans l'espace de phase. Dès lors, on peut accéder toutes les fonctions de distribution: un corps, deux corps,... on n'est limité que par la statistique et done par le coot des calculs.

Les conditions de validité de la C.I. (régime des eollisions) demandent que les collisions binaires soient suffisamment distinctes dans le temps et dans l'espace, ou simplement ( $\mathrm{r}_{\mathrm{s}}=$ portée des forces nucléaires)

$$
r_{s}^{3} \rho \ll 1
$$

Cette condition n'est que marginalement vérifiée encore une fois. De plus, les effets de cohérence ou de champ moyen sont négligés, ce qui à nouveau requiert

$$
\frac{M^{2}\langle q\rangle^{2}}{2 m} \ll \frac{B}{A}
$$

une condition qui n'est certainement pas vérifiée dans la région des spectateurs.

\subsubsection{Dynemique}

Dans la figure 3.5 , nous montrons l'évolution de la densité baryonique dens le plan de là zéaction pour le système $A u+A U$ a 400 MeV/A. Les propriétés principaler de la réaction sont sensiblement les memes que pour le cas hydrodynamique : il y a une phase de compression dans la region centrale suivie diune decompression très rapide. Il y a cependant des différences. D'abord, la matiere semble déviée moins fort dans la cascade. Ensuite, la C.I. prédit la présence, clairement visible dans la Figure 3.5 , de véritables spectateurs, c'est-à-dire de nucléons ne faisant aucune collision. Cet aspect de la cascade est refleté dans la Figure 3.6, qui montre la fréquence des collisions subies par un nucléon. On voit que quel que soit le système, et même pour les parametres d'impact nuls, il y a toujours des nucléons qui interagissent peu. Las nucleons $n=0$ et $n=1$ sont responsables de l'anisotropie dens le con. de l'émission de nucleons dans les collisions des systemes symetriques ${ }^{11}$.

La Figure 3.7 donne l'évolution du systeme de la figure 3.5 dans l'espace des impuisions. On peut distinguer la fin l'effet de l'emission latérale. 


\subsection{Equation de Landau-Vlassov}

3.4.1. Dérivation

L'équation de Landau-Vlassov a été déduite intuitivement par Landau. Néanmoins, et c'est très utile pédagogiquement, on peut la dériver rapidement de l'équation de Sehrödinger. Du point de vue de la mécanique quantique non relativiste, la description du systeme est fournie par la matrice densité à A corps

$$
\vec{\rho}\left(\vec{I}_{1}, \ldots, \vec{I}_{A}, \vec{I}_{1}, \ldots, \vec{z}_{A}, t\right)=\psi *\left(\vec{I}_{1}, \ldots, \vec{I}_{A}, t\right) \psi\left(\vec{I}_{1}, \ldots, \vec{I}_{A}, t\right),
$$

qui obeit à l'équation de Von Neumann

$$
\frac{\partial \tilde{\rho}}{\partial t}=\frac{\dot{i}}{h}[H, \tilde{\rho}] .
$$

Par integration sur les coordonnés de $A-1, A-2, \ldots$ particules, on peut construire la matrice densité à un corps, à deux corps,

$D^{(1)}(\vec{I}, \vec{I}, t, t)=A \int d^{3} r_{2} \ldots d^{3} r_{A} \tilde{p}\left(\vec{r}_{I}, \vec{r}_{2}, \ldots, \vec{r}_{A}, \vec{r}^{I}, \vec{r}_{2}, \ldots, \vec{r}_{A}, t\right)$,

$\left.\rho^{(2)}\left(\vec{r}_{1}, \vec{r}_{2}, \vec{r}_{1}, \vec{r}_{2}, t\right)=A(A-1) \int d^{3} r_{3} \ldots d^{3} F_{A} \tilde{\rho}_{r_{1}}, \vec{r}_{2}, \vec{r}_{3}, \ldots \vec{r}_{A}, \vec{r}_{1}, \vec{r}_{2}, \vec{r}_{3}, \ldots \vec{r}_{A}, t\right) \ldots$

En faisant les menes intégrations sur ( 3.10$)$, on peut obtenir des équations de mouvement pour $p^{(1)}, \rho^{(2)}, \ldots$ Airisi, pour $p^{(1)}$, on obtient

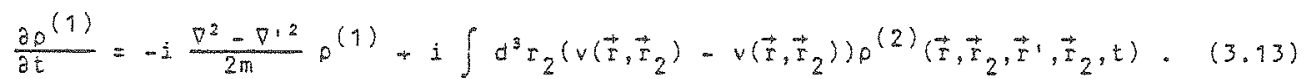

Il est interessant à ce stade o'introduire la transformé de Wigner de la densité à un corps

$$
f_{1}(\vec{I}, \vec{p}, t)=\int \frac{d^{3} s}{(2 \pi h)^{3}} e^{\frac{i}{b} \vec{p} \cdot \vec{s}}(1)\left(\vec{r}+\frac{\vec{s}}{2}, \vec{I}-\frac{\vec{s}}{2}, t\right) \quad .
$$

Cette fonction se comporte a bien des égards comme la fonction de distribution a un cosps (nous utiliserons le meme symbole pour les deux quantités). En particulier, pour les deux quantités, la densité de particules $p$, le courant de particules $\mathrm{j}^{\mathrm{j}}$ et ie tenseur d'impulsion sont donnés per les memes inténrales

$$
\begin{aligned}
& p(\vec{r}, \hat{t})=\int d^{3} p \vec{r}_{1}(\vec{r}, \vec{p}, t) \\
& j(\vec{r}, t)=\int d^{3} p \vec{p} \vec{r}_{1}(\vec{r}, \vec{p}, t)=p(\vec{r}, t) \vec{u}(\vec{I}, t) \\
& \bar{r}_{i j}=\int d^{3} p p_{i} p_{j} \vec{f}_{1}(\vec{I}, \vec{p}, t) .
\end{aligned}
$$




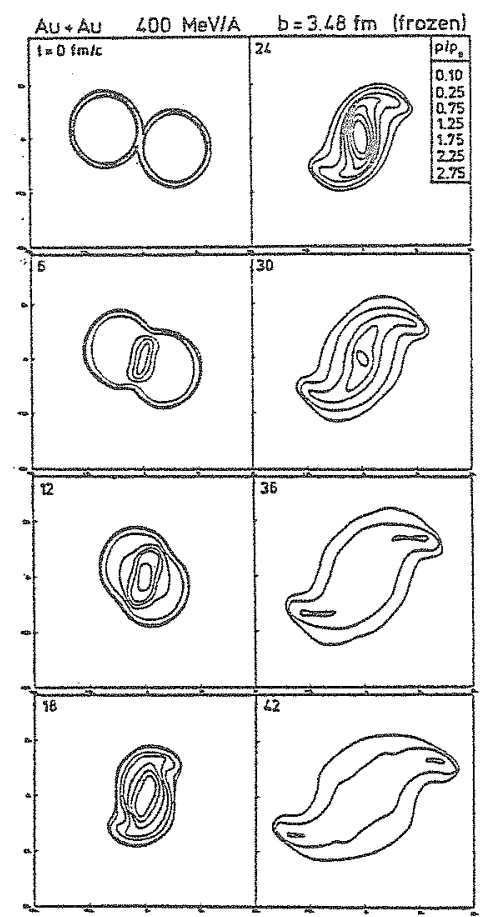

Figure 3.5. Evolution de la densité dans un calcul de C.I. ${ }^{20}$.

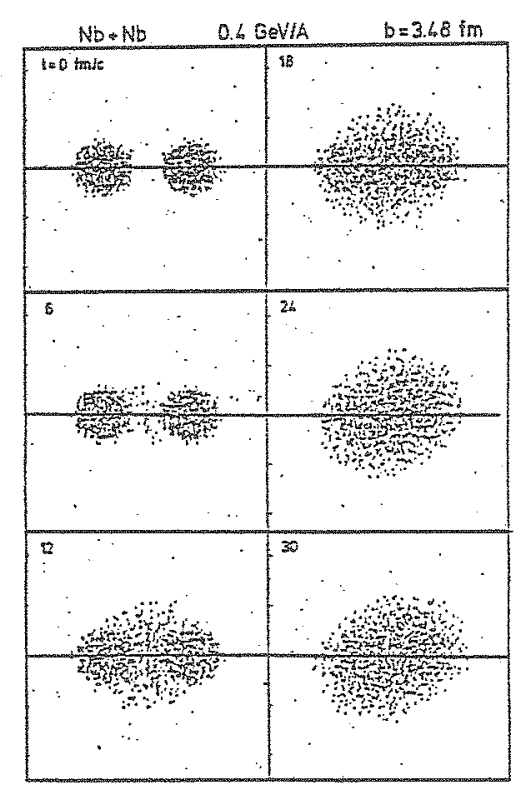

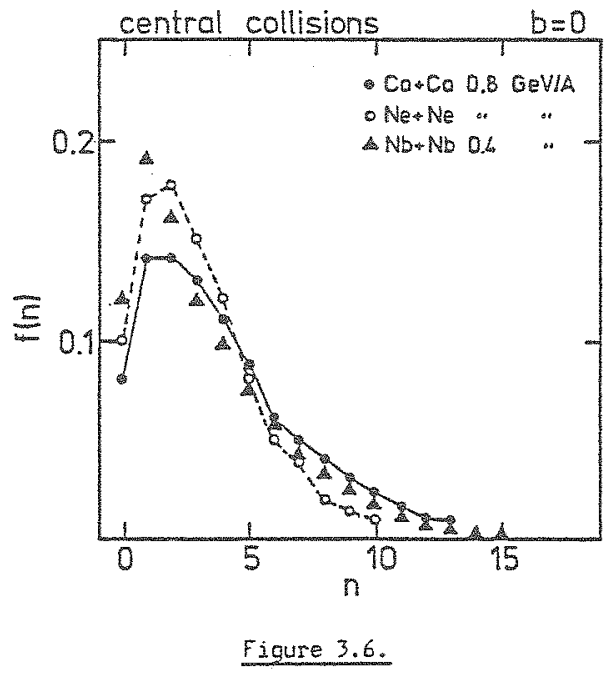

Fréquence du nombre de collisions effectuées

par un nucléon.

\section{Figure 3.7.}

Evolution de la densité dans l'espace des impulsions, d'après un calcul de C.I. ${ }^{20}$. 
En utilisant (3.14), on peut ecrire (3.13) sous la forme

$$
\begin{aligned}
\left(\frac{\partial}{\partial t}+\frac{\vec{p}}{m} \cdot \vec{v}\right) F_{1}= & i \int \frac{d^{3} x}{(2 \pi k)^{3}} e^{\frac{i}{h} \vec{p} \cdot \vec{x}} \int d^{3} x "\left[v\left(\vec{r}+\frac{\vec{x}}{2}, \vec{r}^{\prime \prime}\right)-v\left(\vec{r}-\frac{\vec{x}}{2}, \vec{r}^{\prime \prime}\right)\right] \\
& \times \int d^{3} p_{1} e^{-\frac{i}{h} \vec{p}_{1} \cdot \vec{x}} \int d^{3} p_{2} r_{2}\left(\vec{r}, \vec{r}^{\prime \prime}, \vec{p}_{1}, \vec{p}_{2}, t\right) .
\end{aligned}
$$

On peut considérer les approximations successives de cette équation. La plus simple consiste en la factorisation de $f_{2}$ en un produit de deux $f_{1}$. On obtient ainsi $\left(\frac{\partial}{\partial t}+\frac{\vec{p}}{m} \cdot \vec{b}\right) f_{1}=i \int \frac{d^{3} x}{(2 \pi k)^{3}} \int d^{3} p_{1} e^{\frac{i}{h}\left(\vec{p}-\vec{p}_{1}\right) \cdot \vec{x}}\left[U\left(\vec{r}+\frac{\vec{x}}{2}\right)-U\left(\vec{r}-\frac{\vec{x}}{2}\right)\right] f_{1}\left(\vec{r}_{1} \vec{p}_{1}, t\right)$

$$
U(\vec{r})=\int d^{3} r^{1} d^{3} p^{1} v\left(\vec{r}, \vec{r}^{\prime}\right) f_{1}\left(\vec{r}^{\prime}, \vec{p}^{\prime}, t\right) \quad .
$$

Si l'on suppose que $U$ ne varie pas beaucoup, on peut développer $U$ en série de puissance de $\vec{x}$ dans $(3.19)$. En retenant le premier terme seulement, on a l'équation de Vlassav

$$
\left(\frac{\partial}{\partial t}+\frac{\vec{B}}{m} \cdot \vec{\nabla}-(\vec{\nabla} U) \cdot \overrightarrow{v_{\vec{p}}}\right) f_{1}(\vec{r}, \vec{p}, t)=0
$$

Cette équation a la même forme que l'équation de Liouville pour des particules indépendantes dans un champ de farce $U(\vec{r}, t)$. La différence est qu'ici le champ est self-consistent. De $(3.18)$ à $(3.21)$, on a considéré deux approximations : l'une de champ moyen local, I'autre (coupant les dérivées d'ordre supérieur de u) de mouvement classique. On pourrait parfaitement s'en affranchir, mais cela laisserait l'equation ( 3.21 ) dans la même forme. L'approximation de champ moyen est ici une approximation d'Hartree, car on a négligé la statistique en factorisant. On peut tout systématiser. L'équation d'Hartree-Fack dépendant du temps s'obtient pius facilement sur la matrice densité, en faisant

$\rho^{(2)}\left(\vec{r}_{1}, \vec{r}_{2}, \vec{r}_{1}^{\prime}, \vec{r}_{2}^{\prime}, t\right)=\rho^{(1)}\left(\vec{r}_{1}, \vec{r}_{1}, t\right) \rho^{(1)}\left(\vec{r}_{2}, \vec{r}_{2}^{\prime}, t\right)-\rho^{(1)}\left(\vec{r}_{1}, \vec{r}_{2}^{\prime}, t\right) \rho(1)\left(\vec{r}_{2}, \vec{r}_{1}^{\prime}, t\right)$.

Dans la représentation de Wigner, l'équation correspondant à (3.21) s'écrit alors

$$
\left\{\frac{\partial}{\partial t}+\frac{\vec{p}}{m} \cdot \vec{\nabla}+\frac{2}{\vec{p}} \sin \left\{\frac{W}{2}\left[(\vec{\nabla}, \vec{p}) \cdot \vec{\nabla}-(\vec{\nabla} W) \cdot \vec{\nabla}_{p}\right]\right\}\right\} f_{1}(\vec{r}, \vec{p}, t)=0,
$$

avec

$W(\vec{r}, \vec{p})=\int d^{3} r^{1} d^{3} p^{1} v\left(\vec{r}, \vec{r}^{\prime}\right) r\left(\vec{r}^{\prime}, \vec{p}^{\prime}, t\right)-\int d^{3} S e^{\frac{i}{h} \vec{p} \cdot \vec{S}} v\left(\vec{r}+\frac{\vec{s}}{2}, \vec{r}-\frac{\vec{s}}{2}\right)$

$$
x \int d^{3} p^{\prime} e^{\frac{\dot{i}}{\not{h}} \stackrel{+}{p}^{\prime} \cdot \vec{s}} f_{1}\left(\vec{r}, \vec{p}^{\prime}, t\right)
$$

L'approximation d' Hartree consiste à négliger le second terme de (3.24) et 
1'approximation classique revient à prendre la limite $h \rightarrow 0$. Pour l'instant, rous utiliserons seulement l'approximation (3.21).

L'approximation au-delà du champ moyen s'obtient en utilisant dans (3.18) la première approximation de I'équation similaire pour $f_{2}$. Bien sur, celle-ci fera apparaftre un $f_{1}$ en plus et done deviendra non-linéaire et contiendra un ordre en plus dans linteraction. Nous donnons ici seulement le résultat, qui a été dérivé à l'aide d'un formalisme different, celui des fonctions de Green ${ }^{21}$ :

$$
\begin{aligned}
& \left(\frac{\partial}{\partial t}+\frac{\vec{P}}{m} \cdot \vec{\nabla}-(\vec{\nabla} U) \cdot \vec{\nabla}_{\vec{p}}\right) f_{1}=\int \frac{d^{3} p_{2}}{(2 \pi)^{3}} \frac{d^{3} p_{3}}{(2 \pi)^{3}} \frac{d^{3} p_{4}}{(2 \pi)^{3}} W\left(\vec{p}_{3} \vec{p}_{4} \rightarrow \vec{p}_{2}\right) \\
& \times \delta^{3}(\vec{p}) \delta(E)\left[\tilde{f}_{3} \widetilde{f}_{4}(1-\tilde{f})\left(1-\tilde{f}_{2}\right)-\tilde{f}_{2}\left(1-\tilde{f}_{3}\right)\left(1-\tilde{f}_{4}\right], \quad 3.25\right)
\end{aligned}
$$

avec

$$
\tilde{f}_{i}=f_{1}\left(\vec{I}, \vec{p}_{i}, t\right)(2 \pi k)^{3} \quad .
$$

Dans cette équation, connue sous le nom de Landau-Valssor (LV), West le taux de colisions conduisant deux particules d'impulsion $\vec{p}_{3}, \vec{p}_{4}$ vers des tats dimpulsion $\vec{p}_{1}, \vec{p}_{2}$. Les fonctions delta expriment les lois de conservation. Enfin, les facteurs $1-\tilde{f}$ tiennent compte de la statistique de Fermi.

Dans une théorie perturbative, $U$ est donné comme ci-dessus et $W$ devient

$$
W\left(p_{3} p_{4}+P P_{2}\right)=\frac{1}{2}\left[v\left(\vec{p}, \vec{p}_{3}\right)-v\left(\vec{p}_{2}, \vec{p}_{4}\right)\right]^{2},
$$

où lion a introduit le potentiel d'interaction en représentetion $\vec{p}$.

Dans le cas nucléaire (a haute énergie), le potentiel est singulier, et un développement en série de puissances de $\checkmark$ n'est pas très utile. L"ergenent habituel est qu'une paire de nucléns sont diffusés un grand nambre de fois ghat que l'un dentre eux interagisse avec un troisième. Dans cette limite, il est plus indiqué de prendre $U=0$ et

$$
W\left(p_{3} p_{4} \rightarrow p p_{2}\right) \delta(E)=\left|\left\langle\vec{p}_{3} \vec{p}_{4}|T| \vec{p}_{2}\right\rangle\right|^{2} \delta(E)=8 \pi^{2} \sigma v_{12} \delta\left(p_{12}-p_{34}\right)
$$

où $T$ est la matrice de transition (libre) habituelle et oú $\mathrm{V}_{12}$ est la vitesse re lative. Cependant, on ne peut négliger les effets de champ moyen, ainsi que nous l'avons vu. Par conséquent, nous postulerons sans plus de justification, une équation du type (3.25) aver (3.28), laissant en suspens la definition exacte de $U(\vec{r})$. L'équation de LV contient un terme de collision binaire. On pourrait être tenté de dire que la C.I. revient à négliger U dans LV. Cependant, il convient de remarquer que la C.I. correspond à un terme de collision plus général que l'équation de LV. Dans ce cas, aucune hypothèse sur la forme de $f_{2}$ n'est requise. De plus, la C.I. peut faire des predictions sur les fonctions de distribution dordre plus élevé.

L'équation de LV ne fait aucune hypothèse sur l'équilibre local. De plus, elle introduit l'energie d'interaction, sous une forme simplifiée sans doute, d'un champ self-consistent. Il est done permis de penser que l'équation de LV contient l'hydrodynamique sous certaines limites (ce que nous verrons plus iain). A part 
les restrictions émises, l'équation de LV constitue donc un cadre tout indique pour comparer les deux théories.

\subsubsection{Moments}

Il est très inéressant de regarder les premiers moments en p de l'équation de LV. Le moment d'ordre zéro donne l'équation de continuité (grâce à (3.15)-(3.16)

$$
\frac{\partial \rho}{\partial t}+\vec{\nabla} \cdot(\rho \vec{u})=0 \quad .
$$

Le premier moment donne (grâce à $(3.15)-(3.17)$ et $(3.29)$ )

$$
\rho\left[\frac{\partial \vec{u}}{\partial t}+\vec{u} \cdot \vec{\nabla} \vec{u}\right]+\vec{\nabla} \cdot \underset{\sim}{\sim}=0 \quad,
$$

avec

$$
\Pi_{i j}=s_{i j}+\delta_{i j}(\rho U-V)
$$

et

$$
V(\rho)=\int_{0}^{p} U\left(0^{\prime}\right) d p^{\prime}
$$

Le tenseur $S_{i j}$, appelé tenseur des tensions, est obtenu en faisant la décomposition

$$
\vec{p}=m \vec{u}+\delta \vec{p}
$$

dans l'expression (3.17) du tenseur des impulsions $\tau_{i j}$. On a

$$
\tau_{i j}=R_{i j}+S_{i j}
$$

avec

$$
R_{i j}=\rho u_{i} u_{j}
$$

et

$$
S_{i j}=\int d^{3} p(\delta \vec{p})_{i}(\delta \vec{p})_{j} f_{1}(\vec{r} \vec{p}, t)
$$

Arrétons-nous un instant sur la signification physique de l'équation (3.30). Elle indique comment l'impulsion est transférée du mouvement macroscopique vers les degrés de liberté microscopiques et inversément. S'il y a des tensions dans le fluide $\left(\pi_{i j} \neq 0\right)$, cela générera un écoulement. Les tensions sant de deux types: elles proviennent de l'agitation autour du mouvement macroscopique $\left(\mathrm{s}_{1 j}\right)$ et de l'énergie accumulée par la compression (second terme de (3.31)).

Pour écrire l'équation pour le second moment, nous introduisons la décomposition suivante : 


$$
s_{i j}=\frac{1}{3} \operatorname{tr} S \delta_{i j}+s_{i j}^{0}=\tilde{p} \delta_{i j}+s_{i j}^{0},
$$

ou le tenseur de trace nulle $5^{\circ}$ est appelé le déviateur. On peut alors dériver les équations suivantes:

$$
\begin{aligned}
& \frac{\partial R_{i j}}{\partial t}+\vec{\nabla}_{0}\left(\vec{u} R_{i j}\right)=-u_{i} \nabla_{j} \tilde{p}-u_{j} \nabla_{i} \tilde{p}-u_{i} \sum_{k} \nabla_{k} S_{k j}^{0}-u_{j} \sum_{k} \nabla_{k} s_{k i}^{0} \\
& \frac{\partial \bar{p}}{\partial t}+\vec{\nabla} \cdot(\vec{p} \vec{u})=-\frac{2}{3} \bar{p}(\vec{\nabla} \cdot \vec{u})-\frac{1}{3} \sum_{i} \sum_{k} S_{i k}^{0}(\vec{\nabla} \vec{u})_{k i}^{S}-\frac{2}{3} \vec{\nabla} \cdot \overrightarrow{3} \\
& \frac{\partial}{\partial t} s_{i j}^{D}+\vec{\nabla} \cdot\left(s_{i j}^{D} \vec{u}\right)=-\frac{2}{3} s_{i j}^{0} \vec{\nabla} \cdot \vec{u}-\vec{\nabla} \cdot \int d^{3} \vec{p}\left[\delta p_{i} \delta p_{j}-\frac{1}{3}(\delta p)^{2} \delta_{i j}\right] \vec{p} f_{1} \\
& +\vec{p}(\vec{\nabla} \cdot \vec{u})_{i j}^{5}-\frac{1}{2}\left[S^{0}:(\vec{\nabla} \vec{u})^{S}\right]_{i j}^{S}-\frac{1}{2} \sum_{k}\left[S_{j k}^{0}(\overrightarrow{\nabla u})_{k i}^{A}=S_{i k}^{0}(\overrightarrow{\nabla u})_{k j}^{A}\right] \\
& +\int\left[\delta p_{i} \delta p_{j}-\frac{1}{3}(\delta \vec{p})^{2} \delta \delta_{i j}\right] i
\end{aligned}
$$

Dans ces équations, I représente le terme de collision dans (3.25), $\tilde{\rho}$ vaut

$$
\tilde{p}=\bar{p}+(\rho U-v)
$$

$(\overrightarrow{\nabla u})^{5}$ est le tenseur symétrique de trace nulle

$$
(\vec{\nabla} \vec{u})_{i j}^{S}=\nabla_{i} u_{j}+\nabla_{j} u_{i}-\frac{2}{3} \delta_{i j} \vec{\nabla}_{0} \cdot \vec{u},
$$

$(\overrightarrow{\nabla u})^{A}$ est le tenseur antisymétrique

$$
(\vec{\nabla} \vec{u})_{i j}^{A}=\nabla_{i} u_{j}-\nabla_{j} u_{i}
$$

J est le courant d'energie (cinêtique interne)

$$
\vec{J}=\frac{1}{2} \int d^{3} p \vec{p} p^{2} \tau_{1}(\vec{r}, \vec{p}, t) \quad,
$$

et $[A: B]^{5}$ est le tenseur symetrique construit à partir de la contraction de $A$ et de $B$.

L'équation (3.38) est strictement équivalente à l'équation du premier moment (3.30). L'équation $(3.39)$ peut être modifiée en utilisant $(3.41)$ et le fait que $\overrightarrow{\nabla V}=\frac{\partial \dot{V}}{\partial \rho} \cdot \vec{\nabla} \rho$. On obtient, en utilisant

$$
\begin{gathered}
u=\frac{3}{2} \vec{p}+v \\
\frac{\partial u}{\partial t}+\vec{\nabla} \cdot(u \vec{u})=-\vec{p} \vec{\nabla} \cdot \vec{u}-\frac{1}{2} \sum_{i} \sum_{k} s_{i k}^{0}(\vec{\nabla} \vec{u})_{k i}^{s}-\vec{\nabla} \cdot \vec{J}
\end{gathered}
$$

Iei, u joue le rôle d'une densité d'énergie (voir (2.8)). Nous y reviendrons plus loin.

Pour toutes rébarbatives que les équations (3.38)-(3.40) puissent parâtre, elles nien ont pas moins une interprétation physique toute simple, si nous 
anticipons sur le fait qu'a l'équilibre p représente la pression et que le déviateur indique justement les tensions hors équilibre. Ainsi, l'équation (3.38)

indique que I! ́́volution de l'écoulement résulte du travail des pressions et de celui des tensions hors équilibre. L'équation (3.39) traduit l'influence de la divergence des vitesses (reliée en quelque sarte à la variation de densité), des tensions hors équilibre et du flux de tension (J) sur l'evolution de la pression. Enfin, I'équation (3.40) danne la variation des tensions hors équilibre. On peut distinguer des formes de "self-interaction", un terme venant du courant des tensions (intégrale sur $f_{1}$ ) et le terme de collisions. On peut s'attendre à ce que celui-ci ait tendance à diminuer les tensions. Enfin, il y a une "rétroaction" de la pression sur la variation du déviateur.

L'equation (3.46) a une interprétation plus simple encore. La variation d'énergie interne vient du travail lié a la compression ou la dilatation du systeme $(\vec{\nabla} \cdot \vec{u})$, des tensions hors équilibre et du transport de l'énergie thermique : $\vec{j}$ représente le flux de chaleur.

Les équations $(3.29),(3.30),(3.39)$ et $(3.40)$ ne sont pas équivalentes l'équation de LV. Les premiers moments d'une fonction constituent une description très reatreinte de la fanction. En fait, ces équations ne peuvent etre résolues en tant que telles. Cela nécessiterait la connaissance du terme de collision dans (3.40). Néanmoins, elles sont très utiles pour étudier certaines limites et pour discuter les problèmes d'équilibre thermodynamique.

Si dens le premier membre de l'équation de LV on avait gardé les termes de Hartree-fock généraux (Equation (3.21)), les équations des moments contiendraient des termes supplémentaires dont l'interprétation n'est pas aisée 22 .

\section{THERMALISATION ET THERMODYNAMIQUE}

\subsection{Introduction}

Dans ce chapitre, nous tenterons de répondre à deux questions que bien des expérimentateurs se posent tous les jours. Peut-on parler de température lorsque l'on observe un spectre experimental exponentiel ? Peut-on utiliser des concepts thermodynamiques pour discuter la dynamique diune réaction ? Une autre question vient en corollaire: l'observation d'un spectre exponentiel est-il indicatif d'un système thermalisé ?

Pour montrer que la prudence doit être de riqueur lorsque l'on analyse un spectre expérimental exponentiel, nous reproduisons la figure 4.1. Celle-ci montre qu'un spectre exponentiel peut provenir de la superposition de différentes sources vant des caracteres différents. Cet exemple est tiré d'un modele théarique, extreme il est vrai, mais néanmoins indique le genre d'interpretations fausses qu peuvent arriver. Il faut ajouter à cela que, en général, on somme sur beaucoup de parametres d'impact, ce qui à nouveau peut correspondre à autant de sources différentes.

Iei cependant, nous voudrions réfléchir sur plusieurs cas concrets qui se pretent à des réflexions theoriques et en tirer des indications pour l'analyse expérimentale. On peut d’abord se demander ce qui caractérise un systeme à 
1'equilibre. C'est ce que nous allons discuter dans la section suivante, en distinguent équilibre global et équilibre local.

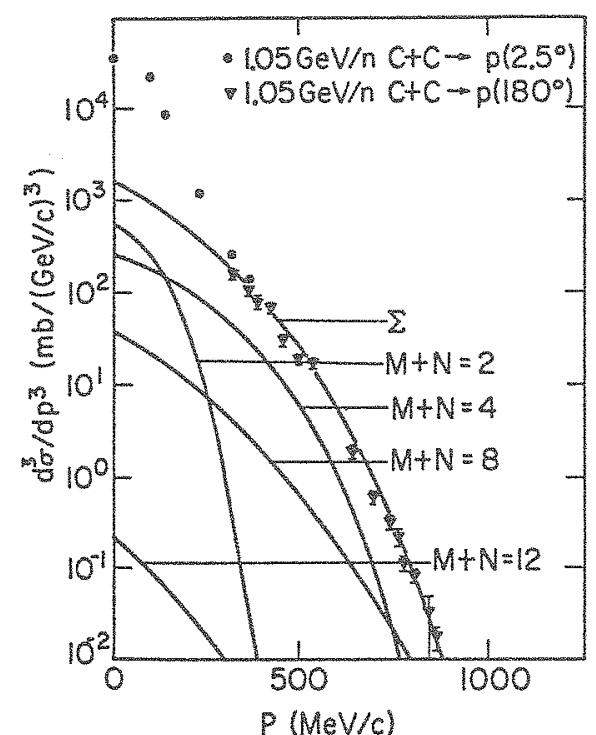

Figure 4.1

Spectre des protons émis dans la réaction indiquéé, décomposée sur le modèle des clusters interagissant ${ }^{23}$.

\subsection{Equilibre global. Equilibre local}

Nous sommes familiarisés avec l'équilibre d'un systeme fluide macroscopique confiné dans un volume fini. S'il n'y a pas de fores à lonque portée, l'équilibre est caractérisé par l'uniformité des variables thermodynamiques $p$, $T$ (et les autre par voie de conséquence). Si l'on se tourne vers une description microscopique, un systeme (de particules de Boltzmann) à l'équilibre se caractérise par une distríbution à un corps du type

$$
f(\vec{r}, \vec{p})=\rho g(\varepsilon) e^{-\frac{\varepsilon(p)}{k T}},
$$

où $\varepsilon(p)$ est l'énergie à une particule pour l'impulsion pet où $q(\varepsilon)$ est la densité d'états d'énergiee. L'équilibre est également caractérisé (pour des interactions isotropes et statiques) par l'absence de corrélations dans l'espace des impulsions

$$
f_{2}\left(\vec{I}_{2}, \vec{p}_{1}, \vec{I}_{2}, \vec{p}_{2}\right)=g\left(\left|\vec{I}_{1}-\vec{I}_{2}\right|\right) F\left(p_{1}\right) F\left(p_{2}\right) \quad,
$$

où $F$ est la distribution dans l'espace des impulsions. La fonction de corrélation $g(r)$ représente la probabilité pour qu'une particule se trouve à une distance $\Sigma$ d'une autre. Pour un gaz dilué (gaz parfait), on a $E(p)=p^{2} / 2 m$ et $g(r)=1$ a ce qui dit que 1'on peut trouver une particule à n'importe quelle distance d'une autre. Les déviations vis-à-vis de cela indique les effets des interactions. Dans un liquide, par exemple, $g(r)$ présente qénéralement un maximum à une certaine 
valeur de $r$. La fonction $g(r) \rightarrow 1$ très rapidement, après une distance qui est typiquement de l'ordre de la portée des forces. L'équilibre est également caracterisé par d'autres grandeurs, que nous ne considérerons pas ici. Attirons cependant l'attention sur le fait qu'un systeme à l'equilibre ne contient aucune corrélation dans l'espace de phase. C'est-à-dire que l'on doit avoir $(i, j=1,3)$

$$
\int r_{i} p_{j} f_{i}(\vec{r}, \vec{p}) d^{3} r d^{3} p=0 \quad,
$$

si $\vec{I}_{i}$ représente la position à partir du centre de masse. L'équation (4.1) vérifie Eutomatiquement $(4.3)$.

Dans les collisions entre ions lourds, le système n'a pas une densité homogène et (4.1) n'est certainement pas valable. On ménage alors la possibilite d'avoir un équilibre local (dans l'espace et dans le temps). Cet équilibre est caractérisé par une fonction de distribution à un corps

$$
f_{1}(\vec{r}, \vec{p}, t)=\rho(\vec{r}, t) g(\varepsilon) e^{-\frac{\varepsilon[\vec{p}-m \vec{u}(\vec{r}, t)]}{k T(\vec{r}, t)}}
$$

et par une fonction de distribution à deux corps exprimé dans le référentiel ou la matière est au repos du type (4.2), avec cette fois des grandeurs qui varient dans l'espace-temps. Si nous considérons le formalisme de la section 3.4, un équilibre local est caractérisé par

$$
I=0, \quad S_{i j}^{0}=0,
$$

'est-à-dire par l'annulation ou terme de collision et par l'annulation du déviateur ( $f$ ). Remarquans qu'en principe l'annulation du déviateur est beaucoup moins restrictive que la condition $(4.4)$.

11 apparaft opportun de discuter ici une objection souvent entendue. Supposons que (4.5) soit réalisée. Souvent, la question suivante est soulevée : comment peut-on appliquer les concepts thermodynamiques à une petite cellule d'un noyau, qui ne contient que deux ou trois nucléons, par exemple? Tant que les conditions (4.5) sont réalisées, on peut considérer que cette petite cellule est en equilibre avec le reste du système. Elle peut donc être décrite par l'ensemble (grand) cannonique dont les prédictions moyennes ne dépendent absolument pas du nombre de particules présentes. Donc, tant qu'on se limitera aux propriétés à un corps, nécessairement sommées sur un grand nombre d'événements, on peut appliquer des considérations thermodynamiques. Si, maintenant, on veut aller au-delà, en étudiant corrélations ou fluctuations, il faut s'attendre à ce que la thermodynamique appliquée à la lettre conduise à des aberrations.

Une restriction cependant : comme un noyau est un systeme fini, il est sujet a des contraintes de conservation de nombres quantiques, en particulier de l'energie. 5i l'énergie d'un système d'un petit nambre de particules est fixée, la distribution (même dans un gaz parfait) ne sera pas une loj de Boltzmann, telle $(4.3)$, ainsi que 1 'indique 1 a Fiqure 4.2 .

(t) Nous verrons cependant, un peu plus tard, une condition un peu moins forte. 


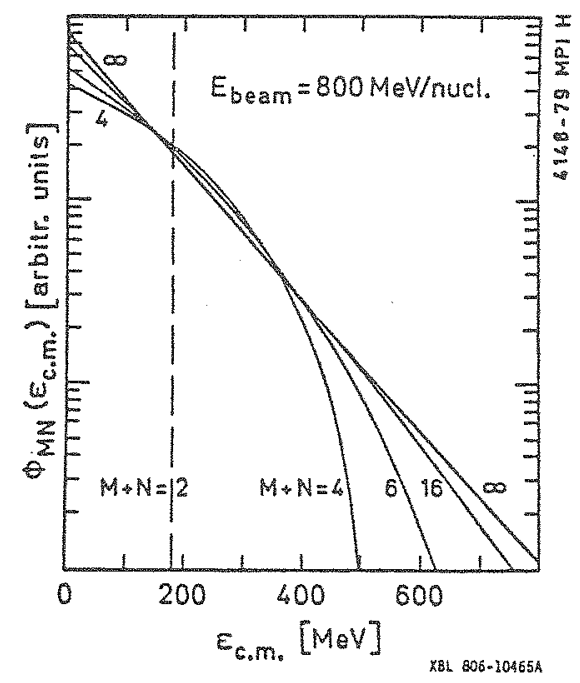

\section{Figure 4.2}

Distribution de l'énergie à une particule pour un système de M+N nucléons dont l'énergie par particule est fixée. Tirée de la Référence 24.

En conclusion, si la condition (4.5) est remplie, on pourra utiliser les concepts thermodynamiques, en n'oubliant pas que l'on fait une thermodynamique simplifíe négligeant les effets liés aux moments élevés de la distribution.f. Nous verrons cependant que $(4,5)$ n'est pas facilement satisfaite.

\subsection{Equilibre local et hydrodynamique}

5i lion a un équilibre local, les conditions (4.5), auxquelles il faut adjoindre $3=0$, sont vérifiées. Les équations $(3.30),(3.38)$ et (3.46) deviennent

$$
\begin{gathered}
\partial\left[\frac{\partial}{\partial t}+\vec{u} \cdot \vec{\nabla}\right] \vec{u}=-\vec{\nabla} \tilde{p} \\
\frac{\partial R_{i j}}{\partial t}+\vec{p} \cdot\left(R_{i j} \vec{u}\right)=-u_{i} \nabla_{j} \tilde{p}-u_{j} \vec{\nabla}_{i} \tilde{p},
\end{gathered}
$$

qui, rappelons-le, est équivalente à la précédente, et

$$
\frac{\partial u}{\partial t}+\vec{\nabla} \cdot(u \vec{u})=-\tilde{p} \vec{\nabla} \cdot \vec{u}
$$

On remarquera que les équations (4.6) et (4.8) sont semblables aux équations (3.2), (3.3) de l'hydrodynamique non-visqueuse $(n=0)$, sauf que (4.8) est écrite pour la densité d'énergie plutot que pour la température. Cependant, à ce stade, Fien ne dit que $\tilde{p}$ est assimilé à la pression ordinaire. En hydrodynamique, on

fait donc une hypothèse supplémentaire qui consiste à supposer que la relation qui relie $u, \tilde{p}$ et $p$ est la même que pour un système macroscopique à l'équilibre.

L'hydrodynamique visqueuse s'obtient en gardant les memes hypothèses, mais en introduisant des corrections, qui bien sur demanderont que $S_{i j}^{0}$ et $J$ soient maintenant différents de zéro. La déviation "minimale" veut que ces quantités soient des fonctions linéaires des gradients des grandeurs intensives décrivant 
l'etat thermodynamique du système (en général, on choisit $\rho, \vec{u}$ et $T$ ). Dès lors, la forme de $5_{i j}^{0}$ et $\vec{J}$ est fixée par leur caractère tensoriel :

$$
S_{i j}^{0}=-n(\vec{\nabla} \vec{u})_{i j}^{S}-\zeta \delta_{i j} \vec{\nabla} \cdot \vec{u} \quad,
$$

où la partie antisymétrique n'a pas été retenue, parce qu'elle violerait l'invariance par réflexion autour d'un centre. Les coefficients $n$ et $\zeta$ sont appelés coefficients de viscosité de cisaillement et de volume (bulk), respectivement. Le vecteur jócrit

$$
\vec{J}=-\lambda \vec{\nabla} \rho-x \vec{\nabla} T,
$$

où $x$ est le coefficient de conductibilite thermique.

On a introduit à dessein des signes négatifs dans les équations (4.9) et ( (4.10), car le second principe de thermodynamique, qui veut que l'entropie d'un système isolé ne peut aller qu'en augmentant, demande $n, b, x \geq 0$ et $\lambda=0$. Ces coefficients sont appelés coefficients de transport, et caractérisent les propriétés du fluide dans des situations de déviations faibles vis-à-vis de l'équilibre. En introduisant ( 4.9$)$ et $(4.10)$ dans les équations $(3.38)-(3.46)$, on retrauve les équations de l'hydrodynamique visqueuse. En général, $\zeta$ est très petit.

\subsection{Expansion et break-up}

Supposons que l'on ait créé un système nucleaire caractérisé par (4.3), c'est-à-dire un équilibre local, ou mêre pour simplifier la discussion par

$$
r_{1}(\vec{r}, \vec{p})=\rho(\vec{r}) e^{-\frac{\varepsilon(\vec{p})}{k T}} .
$$

II ya de soi qu'un tel systeme ne restera pas tel quel. Il va se dilater ou se contracter suivant la valeur de la pression. Aux énergies relativistes, la pression est positive et le système se décomprime rapidement.

Il est intéressant d'etudier ce qui va se passer dans ce cas. Tout en se dilatant, le système est le siège de callisions nucléon-nucléon. A partir d'un certain moment, le systeme sera suffisamment dilué pour que les particules n'entrent plus en collision: c'est le freeze-out. Au dela, c'est une expansion libre: les nucléons s'écartent les uns des autres sans modification de leur mouvement rectiligne.

Supposons maintenant que l'expansion avant le freeze-out soit telle que l'équilibre local soit préservé. Dès lors, l'expansion du système est régie par les équations (3.1), $(4.6)-(4.8)$, où nous pouvans remplacer $\tilde{p}$ par $p$ et par l'équation d'état. Ce probleme a été résolu pour l'expansion radiale d'une boule de gaz parfajt ${ }^{25}$. Les résultats sont assez compliqués, mais on peut se rabattre sur un modele approché ${ }^{26}$, qui suppose que la densité est uniforme à tout moment. Dans ce eas, a cause de ( 3.1 ), le champ des vitesses est

$$
\vec{u}(\vec{r})=-\frac{1}{3} \frac{\dot{\rho}}{\rho} \vec{r}=a \vec{r}
$$


et le spectre d'énergie des nucléons est

$$
f(\varepsilon)-\int_{0}^{1} x^{3} e^{-\left(\varepsilon+\frac{1}{2} m \frac{a^{2} x^{2}}{R^{2}}\right) / k T} \sinh \left(\frac{2 \sqrt{\varepsilon m a^{2} x^{2} / 2}}{R k T} d x, \quad\right. \text { (4.13) }
$$

où $R$ et $Y$ sont le rayon et la température instantanée. Pour faire ressortir les

faits essentiels, nous supposerons comme dans la Référence 27 que la vitesse d'expansion est la même $u_{e}$ partout. Dans ce cas

$$
f(\varepsilon) \sim e^{-\left(\varepsilon+\frac{1}{2} m u_{e}^{2}\right) / k T_{f o}} \sinh \left(\frac{2 \sqrt{\epsilon m u_{e}^{2} / 2}}{k T_{f o}}\right),
$$

ou $T_{f o}$ est la température au monent du freeze-out. Il faut d'abord remarquer que la distribution d'énergie est distordue à cause de l'expansion, ainsi que l'indique la courbe pour les protons de la figure 4.3.

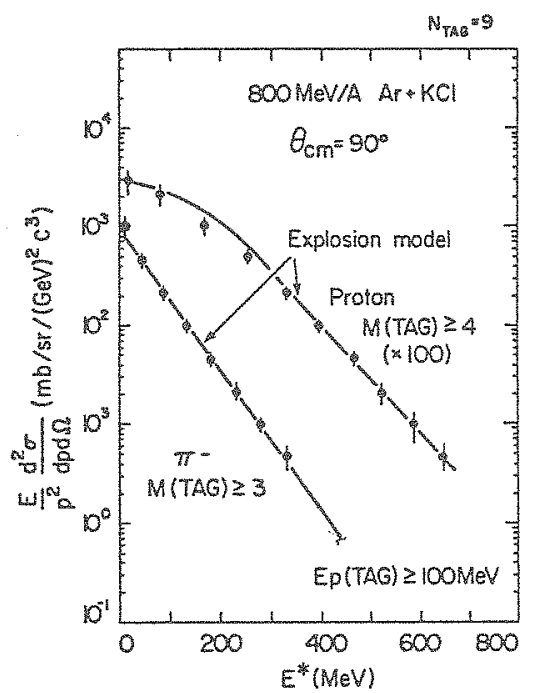

\section{Figure 4.3}

Analyse du spectre des pions et des protons à l'aide de I'équation (4.14). Tiré de la Référence 27.

La température apparente $T^{*}$ tirée de la queue du spectre est différente de Tfo.

on peut le voir facilement, car si $\varepsilon$ est suffisamment grand, on aura

$$
f(\varepsilon) \sim e^{-\frac{\varepsilon}{k T_{f o}}} e^{\frac{2 \sqrt{\varepsilon m u_{e}^{2} / 2}}{k T}}
$$

et en faisant un développement des exposants au premier ordre autour de $\varepsilon_{0}$,

valeur typique de la région que l'on explore:

$$
f(\varepsilon) \sim \varepsilon^{-\frac{\left(\varepsilon-\varepsilon_{0}\right)}{k T^{*}}}
$$




$$
T^{*}=T\left(1-\sqrt{\frac{m u_{\varepsilon}^{2}}{2 \varepsilon_{0}}}\right)^{-1}
$$

Dans le cas de la Figure 4.3, T vaut $60 \mathrm{MeV}, u_{e} \approx 0.3 \mathrm{c}$ et $\gamma * \approx 80 \mathrm{MeV}$.

on peut d'autre part estimer, dans le même cas, la temperature $T$ de la boule de feu initiale. Si l'an néqlige la production de particules, et si lion fait

l'approximation du gaz parfait, la conservation de l'énergie impose

$$
\frac{1}{2} m u_{e}^{2}+\frac{3}{2} k T_{f o}=\frac{3}{2} k T,
$$

ce qui, dans le cas concret ci-dessus donne $T=88$ MeV. La proximité est sans

doute ici accidentelle. Il n'en reste pas moins vrai que la temperature apparente est une fonction monotone de l'énergie d'excitation.

L'expansion gouvernée par les équations (3.1), (4.6), (4.8) est une expansion isoentropique : elle conserve l'entropie. Au freezerout, ces équations cessent d'etre valabies, car le terme de collision disparaft. On peut plus ou moins estimer la densité de freeze-out comme étant le moment ou la distance caractéristique entre les nucléons est de l'ordre de la racine carrée de la section efficace divisée par $\pi$

$$
d=\sqrt{\frac{\bar{\sigma}}{\pi}}
$$

où $\bar{\sigma}$ est la section efficace moyenne entrant dane le terme de collision (voir plus loin). On trouve grosso modo $\rho_{\mathrm{fo}} \sim 0.5-1 \rho_{0}$.

Bien sur, il faut s'attendre à ce que le freeze-out ne soit pas instantané. Après le freeze-out, les corrélations $\vec{r}$. $\vec{p}$ croissent continument. Ceci peut se comprendre si l'on considere un système uniforme et les corrélations $\vec{z} . \vec{p}$ qui peuvent s'y produire. La situation est schématisée dans la Figure 4.4 .

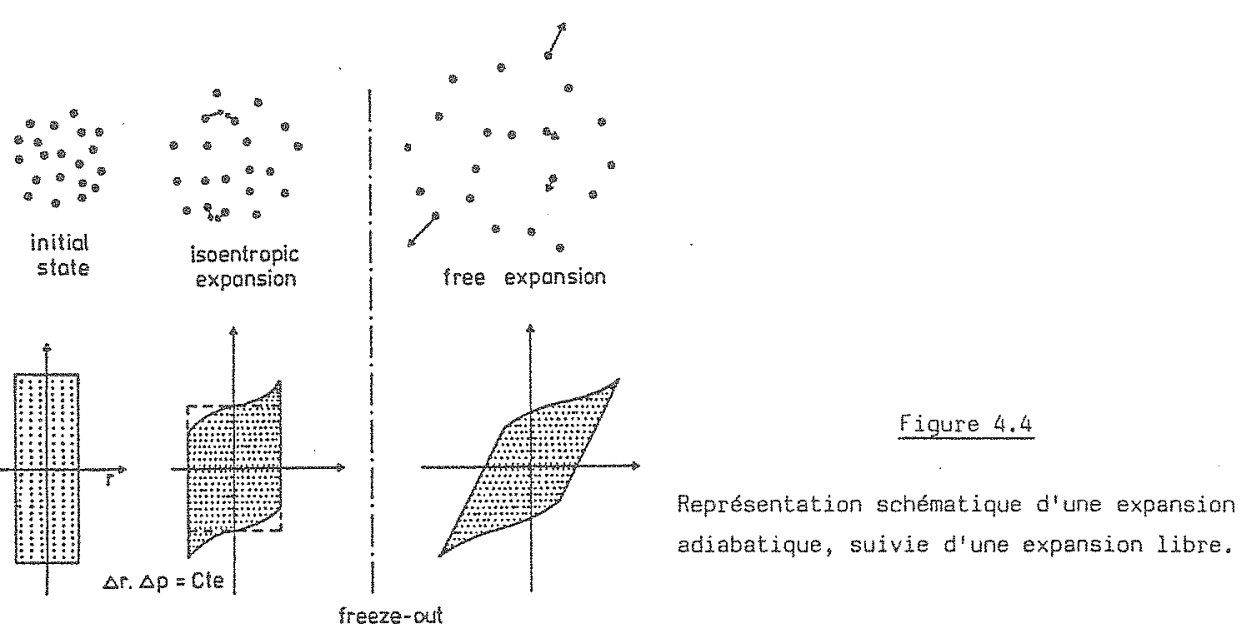


à-dire l'extension du systeme dans l'espace de phase. Si l'expansion se faisait infiniment lentement, le rectangle du bas resterait un rectangle de surface

constante. La vitesse d'expansion doit s'ajouter a la vitesse des particules et déforme ce rectangle. Apres le freeze-out, la vitesse des particules ne change plus, ce qui provoque une homothétie de la figure du bas. Les corrélations $x_{0} p$ vont croitre indéfiniment, ce qui a pour effet que la position et la vitesse

de chacune des particules seront alignées.

\subsection{Ya-t-il Équilibre local?}

Pouvons-nous déterminer l'évalution du déviateur $S_{i j}^{0}$ à partir de l'équation (3.40) ? Une façon d'etudier le problème est de faire une approximation du temps de relaxation pour le terme de collision

$$
\int\left[\delta p_{i} \delta p_{j}-\frac{1}{3}(\delta p)^{3} \delta \delta_{i j}\right] I=-\frac{s_{i j}^{0}}{\tau},
$$

ce qui, par parenthèse,ferme la hiérarchie des équations de moments sur ellesmêmes. Bien sur, (4.20) ne peut étre qu'indicatif, car si l'on a un mouvement le long de l'axe $z$ seulement, comme au début de la collision, $5_{z z}^{0}$ va décraftre, tandis que $S_{x x}^{0}$ va croftre. Le temps de relaxation peut donc se définir par (4.20). on peut l'estimer en utilisant les conditions qui règnent au début de la collision. Ainsi, on a

$$
I=\frac{1}{\rho \sigma \bar{v}} \frac{\left\langle\Delta p_{Z}^{2}\right\rangle}{\left\langle p_{Z}^{2}\right\rangle}=\frac{\lambda}{\bar{v}} f \quad
$$

où $\sigma$ est la section efficace moyenne apparaissant dans le terme de collision, ou $\bar{v}$ est la vitesse relative moyenne et où $\lambda$ est le libre parcours moyen pour faire une collision. Enfin, f représente l'efficacité d'une collision. Il dépend de la section efficace differentielle, qui fait que les particules sant plus ou moins déviées. De l'expérience, on déduit que $f$ décroit quand l'énergie augmente.

A haute énergie, $\tau$ est de l'ordre de 3 à $5 \mathrm{fm} / \mathrm{c}$, alors que le temps de collision est typiquement de 10 à $30 \mathrm{fm} / \mathrm{c}$. Parallèlement, $\lambda_{\text {th }}=\lambda \mathrm{f}$ est de l'ordre de 2 à $5 \mathrm{fm}$, ce qui doit être comparé à la kaille du système, $\sim 10 \mathrm{fm}$. A basse énerqie, les estimations ne sont pas triviales, car il faut tenir compte du principe de Pauli d'une façon adéquate. Celui-ci a pour effet d'inhiber les collisions. Les courbes en traits pleins de la fiqure 4.5 se réfèrent au temps de vie pour une particule traversant un noyau froid. La courbe en trait discontinu correspond à un noyau chaud. On peut voir que cela ne change pas sensiblement les choses. Pour une énergie supérieure a $50 \mathrm{MeV}$, $\tau$ est plus petit que le temps de collision. Le libre parcours moyen a a $50 \mathrm{MeV}$ par nucléon (calculé avec le principe de Pauli) est de l'ordre de $5 \mathrm{fm}$.

on peut ne pas se soucier, dans une large mesure, du temps de collision si l'on considère seulement des moyennes sur l'espace et le temps, avec des intervalles de moyenne de l'ordre de $\lambda$ et $\sigma$, Iespectivement. En effet, toute fluctuation dans $5_{i j}^{0}$ disparastra endéans ces intervalles de temps et despace. En se repartant aux chiffres ci-dessus, il semble bien que l'equilibre local ne peut etre envisagé qu'avec circonspection, à haute aussi bien qu'à basse énergie. 


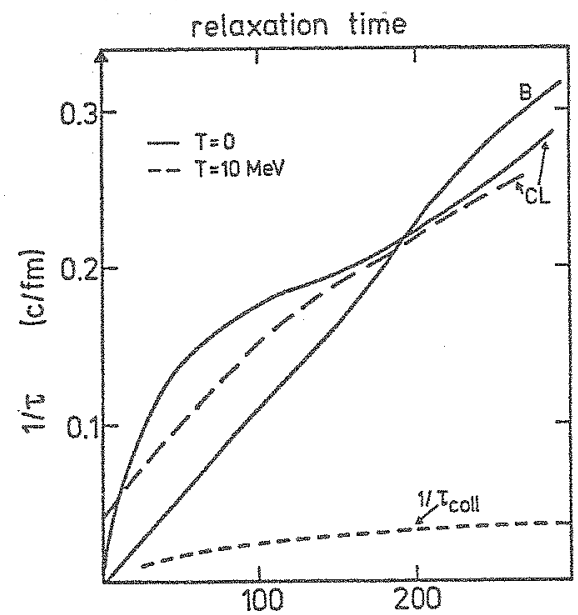

E (MeV)

\section{Figure 4.5}

Temps de relaxation en fonction de l'énergi.e. La courbe $\mathrm{B}$ correspond à un calcul simplifié de la Référence 16, tandis que les courbes $\mathrm{Ci}$ proviennent d'un calcul de Brueckner ${ }^{8}$.

A titre d'illustration, nous donnons dans les Figures $4.6-4.8$ différentes grandeurs intervenant dans la Section 3.4, pour la réaction Au + Au à $400 \mathrm{MeV} / \mathrm{A}$, et ce pour un temps qui correspond grosso modo au temps de compression maximale. La figure 4.6 donne le profil de densité et la distribution du courant de particules. On distingue d'ailieurs trés nettement un "side-splash". La Figure 4.7 donne les contours d'égale pression en MeV $\mathrm{rm}^{-3}$, c'est-à-dire un tiers de la trace du tenseur des tensions $5_{i j}$. La Figure 4.8 décrit schématiquement la distribution du déviateur. En fait, ce qui est porté est la quantité

$$
x=\frac{\sum_{i}\left|s_{i i}\right|}{3 p}
$$

5'il y a équilibre local, $x=0$ et si le mouvement est, dans le référentiel de la matière, uniquement suivant une direction, $x=1$. En drautres termes, les grandes valeurs de x représentent une matière non équilibrée. Il faut remarquer que, sauf pour les régions indiqués par les gros points, le tenseur $5_{i j}$ est toujours caractérisé par $S_{z z}>S_{x x}$, ce qui indique une survivance non négligeable du mouvement initial. De plus, an observe que les éléments non diagonaux de $S_{i j}$ sont un ordre de grandeur plus petits que les éléments diagonaux. D'sprés la Référence 19, ceci signifie que la matière nucléaire (dans ce calcul, tout au moins) se comporte plus comme un fluide ordinaire, ou l'élément dynamique essentiel est la pression, plutot que comme un solide, où l'élément dynamique est le gradient des vitesses. 


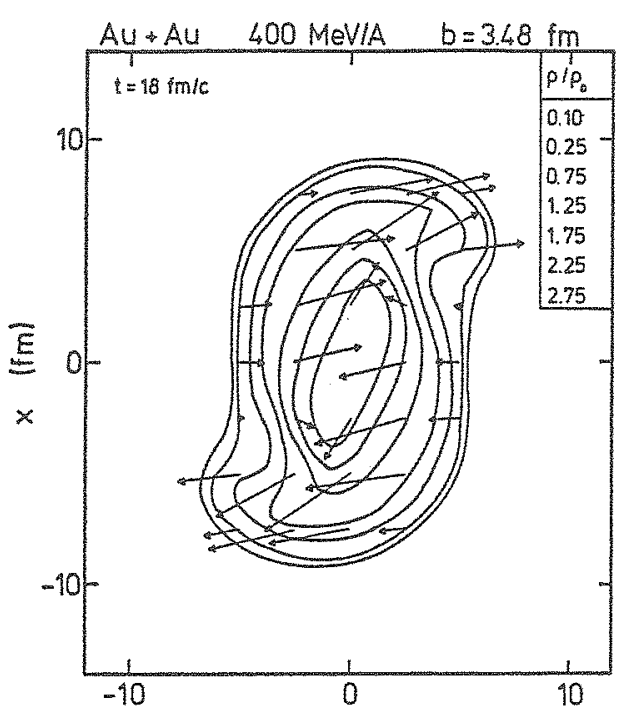

$z$ (im)

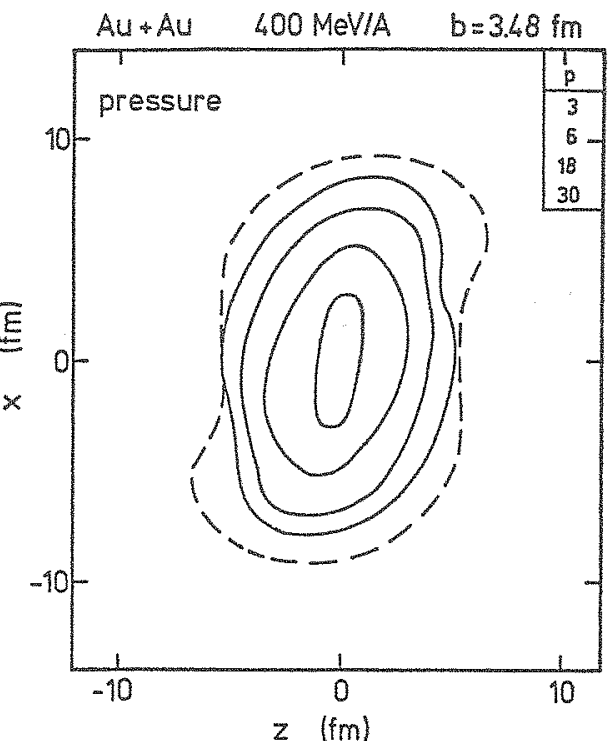

Figure 4.6. Distribution de la densité (courbes) et du courant ( $\vec{j}$ dans l'équation $(3.16)$ ) dans plan de la réaction $\mathrm{Nb}+\mathrm{Nb}$. La grandeur des flèches est proportionnelle à l'intensité du vecteur $\vec{j}$. Tiré de la Référence 20. même réaction que dans $(4.6)$. La courbe en traits discontinus donne la courbe d'isodensité dans la figure précédente. Tiré de la Référence 20.

Même chose que la Figure 4.7 pour la quantité (4.22). Voir texte. Tiré de la Référence 20. 


\section{PROPRIETES DE LA MATIERE DENSE}

\subsection{Considerations théoriques}

Nous allons ici discuter les proprietés de la matière dense formée au cours de la réaction. A ce propos, il est utile d'avoir une sorte de guide, qui nous indique de quelle manière les propriétés de l'état dense dépendent de l'équation d'état et du mécanisme de production.

Pour le premier des points, nous pouvans utiliser l'équation de RankineHuganiot (RH) pour les ondes de choc. Si l'on regarde le haut de la figure 3.3 , on voit que dans le cadre de ce calcul on n'est pas loin d'une configuration plane. En idéalisant les noyaux par des tranches de matière, on peut utiliser l'equation de RH dans la configuration indiquee par la figure 5.1.

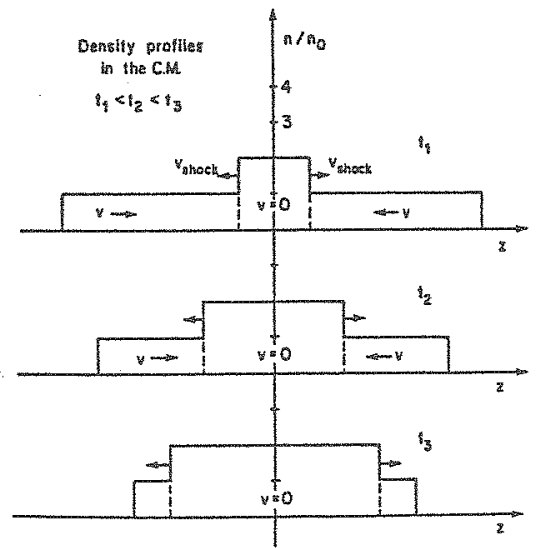

Fiqure 5.1

Profil de densité correspondant à l'apparition d'une onde de choc pendant la collision de deux tranches de matière.

Si on désigne par un indice zéro les quantités avant le choc et sans indice les quantités apress le choc, I'équation de RH s'écrit dans un formalisme non-relativiste

$$
\frac{u}{p}-\frac{u_{0}}{p_{0}}+\frac{1}{2}\left(\frac{1}{p}-\frac{1}{p_{0}}\right)\left(p+p_{0}\right)=0 .
$$

L'équation d'état peut toujours s'écrire comme

$$
u=u(p, p) .
$$

Enfin, nous devons tenir compte de la conservation de l'energie, c'est-à-dire

$$
\frac{u}{p}=\frac{u_{0}}{\rho_{0}}+r_{c m},
$$

où $T_{c m}$ est l'énergie cinétique par nucléon dans le c.m., $u_{0} / \rho_{0}=-16$ MeV et $p_{0}=0.17 \mathrm{fm}^{-3}$. On a trois uations pour trois inconnues. On peut done déterminer les conditions aprés le curc. Ainsi, pour un gaz parfait, on peut montrer que (*) 
La figure 5.2 donne les résuitats d'un tel calcul (relativiste). On peut voir que la pression n'est pas tellement dépendante de l'équation d'état, alors que la den site (et done la température) et, dans une moindre mesure, l'entropie y sont plus sensibles. Il faut cependant remarquer que, de toute manière, une quantité importante d'entropie est produite.

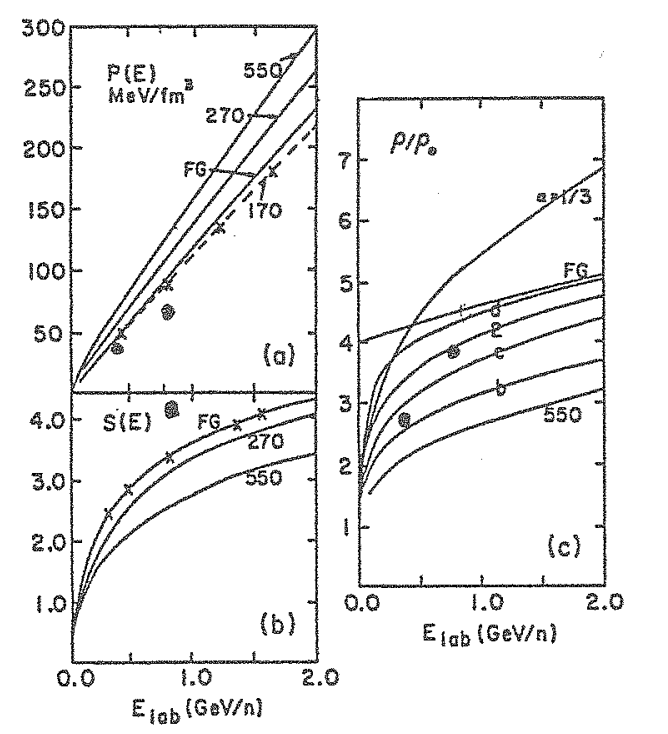

\section{Figure 5.2}

Les courbes donnent les conditions après le choc calculées par l'équation de RankineHugoniot pour differentes équations d'état. La courbe FG correspond au gaz de fermi.

Les gros points donnent le résultat de la C.I. à la compression maximum.

Bien sor, on doit siattendre à ce que le phénomène de compression ne suive pas l'image simpliste de l'onde de choc. Dans la Figure 5.2, on a indiqué quelques résultats obtenus avec un modèle de C.I. L'effet le plus spectaculaire est le fait que la densité y est assez basse et que l'entropie est assez grande. Ce dernier résultat peut provenir de la géométrie qui, contrairement à $R H$, introduit des noyaux finis dans les trais dimensions et aussi à cause de la possibilité de produire des particules $\Delta$. Ceci touche à un autre aspect, qui est la composition "chimique" de la matière dense, que nous ne discuterons cependant pas ici.

\subsection{Expansion et quantités conservées}

Lontemps, on a pensé que la désagrégation du système comprimé formé transitoirement ferait disparaftre les propriétés de cet état dense. Pour prendre une image très prosaique, si l'on observe un ressort détendu, on ne peut dire jusqu'a quelli pression il a été comprimé une heure auparavant, par exemple. Petit à petit, cepel dant, est apparue l'idée qu'il pourrait y avoir des quantités observables qui résistent à l'expansion, c'est-à-dire des quantités dont la valeur est fixée dès le moment de compression maximale. Ainsi, dans la Référence 3 , il est déjà fait mention du fait que le nombre de pions pourrait être une telle grandeur. En fait, 
ainsi que le montre la Figure 5.3, ce qui est conservé par l'expansion est le nombre cumulé de pions et de particules $\Delta$. Cela semble do à une balance délicate en-

tre le temps de vie du $\Delta$ et la vitesse d'expansion.

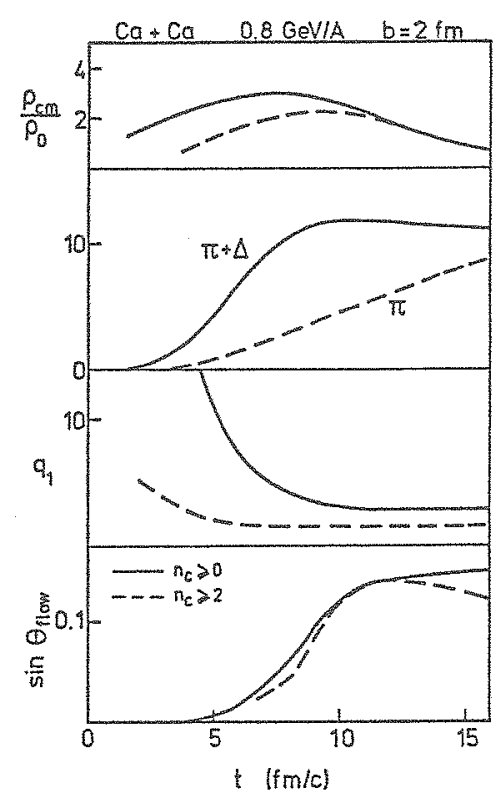

\section{Ficure 5.3}

Evolution dans un modèle de C.I. de diverses quantités lors de la collision Ar + Ca. Pour les ler, उème et 4 ème cadres, les courbes en traits pleins se réfẻrent à tous les nucléons, tandis que pour les courbes en traits discontinus, on n'a pris en compte que les nucléons faisant deux collisions au moins.

Une autre quantité qui est conservée (en grande partie) par l'expansion est I'entropie (Figure 5.4), ainsi qu'il a été démantré pour la première fois dans 1a Référence 5 .

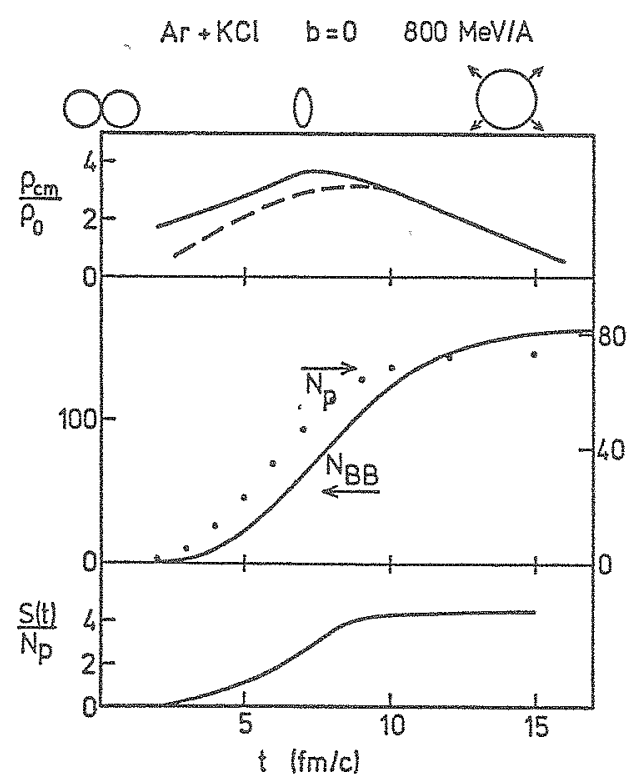

Figure 5.4

Evolution dans un modèle de C.I., de la densité baryonique au $\mathrm{c} . \mathrm{m}$. du système (trait plein : tous les nucléons ; traits discontinus : les participants seulement), du nombre de participants $N_{p}$, du nombre cumulé de collisions baryon-baryon et de l'entropje des nucléons (divisée par le nombre final de participants). 
Enfin, plus récemment, on s'est aperçu que l'angle d'écoulement 20,28 et peut-être aussi la forme du tenseur de sphericite ${ }^{29}$ sont des quantites conservées lors de I'expansion.

Nous reviendrons dans la Section 6 sur les moyens de tirer profit de ces constatations pour retirer des informations sur l'équation d'état.

\subsection{Inage physique de 1'expansion}

Nous avons déja discuté dans la Section 4.4 un modele pour l'expansion, supposant quili siaqit en quelque sorte d'un milieu continu et que plus ou moins obruptement ce milieu se dissout en particules d'une seule espèce. On peut se demander ce qui se passe si l'on va au-delà de ce point de vue. Deux questions peuvent se poser : $Y$ a-t-iI des inhomogénéicés dans le système ? Comment et quand se forment les particules composées que l'on observe expérimentalement?

Il va de soi que la grandeur théorique qui contient les informations nécessaires est la fonction de distribution à deux corps $\vec{f}_{2}\left(\vec{r}_{1}, \vec{p}_{1}, \vec{F}_{2}, \vec{p}_{2}, t\right)$. La connaissance de cette fonction peut dire si à linstant teux particules sont dans un état qui est celui du deutéron, par exemple. Notons que cela ne signifie pas nécessairement que les nucléons ne sont pas corrélés avec un troisième. Pour savoir cela, il faudrait connactre $f_{3}$. On voit donc qu'une thérie complète est très compliquée. Néanmoins, il est intéressant de faire des considérations sur $f_{2}$ En premier lieu, $f_{2}$ obéit une équation d'évolution du type de celle vérifiée par $f_{1}$ (LV). Dans le même genre d'approximation, elie pourrait s'écrire

$\left(\frac{\partial}{\partial t}+p_{1} \cdot \frac{\nabla_{1}}{m}-\vec{\nabla} U\left(r_{1}\right) \cdot \vec{\nabla} p_{1}+p_{2} \cdot \frac{\nabla_{2}}{m}-\vec{\nabla} U\left(\vec{r}_{2}\right) \cdot \vec{\nabla} p_{2}\right) f_{2}\left(\vec{r}_{1}, \vec{p}_{1}, \vec{r}_{2}, \vec{p}_{2}, t\right)$

$\int d^{3} p_{1}^{1} d^{3} p_{2}^{1} d^{3} p_{3}^{2} \int d^{3} p_{3} \delta(\vec{p}) \delta(E) W\left(p_{1} p_{2} p_{3}+p_{1}^{3} p_{2}^{\prime} p_{3}^{1}\right)\left\{f_{2}\left(\vec{r}_{1}, \vec{p}_{1}, \vec{r}_{2}, \vec{p}_{2}, t\right)\right.$

$\left.f_{1}\left(\vec{r}_{3}, \vec{p}_{3}, t\right)-f_{2}\left(\vec{r}_{1}, \vec{p}_{1}, \vec{r}_{2}, \vec{p}_{2}, t\right) \tilde{f}_{3}\left(\vec{r}_{3}, \vec{p}_{3}, t\right)\right\}+$ permo cycliques

+ un terme en $\hat{f}_{3}$.

On voit donc que l'évolution des corrélations du type "cluster" est due à deux choses : d'une part au champ moyen et d'autre part aux collisions. La seconde est facile à comprendre. Pour qu'un deutéron existe en tant que tel, il faut qu'il n'y ait pas trop de nucléns dans le volume compris entre le neutron et le proton. Il va de soit que cela requiere d'etre en-dessous d'une densité critique. A température nulle, cette densité est inférieure à la densité normale. A haute température, la restriction est sans daute maindre. Pour se fixer un guide, on peut admettre que la densité doit rester la même si. I'on remplace des nucléons par des deutérons, ce qui donne $p \sim 0.2 \mathrm{fm}^{-3}$. On peut done admettre que les deutérons et autre clusters apparaissent dans les derniers moments de la réaction.

on peut rapprocher le second facteur d'évolution de $f_{2}$ de ce que lion connast sur $v(r)$ (Eq. (2.24)), qui lui est intimement relie. En effet, une relation thermodynamique (donc yalable à l'écuilibre) bien connue dit que

$$
4 \pi \int_{0}^{\infty} v(r) r^{2} d r=k T\left(\frac{\partial \rho}{\partial p}\right) T-1 \text {, }
$$


ce qui signifie que les corrélations sont conditionnées par le module de campressibilité. La relation (5.6) dit aussi que $V(r)$ doit tendre vers zero tant que l'on est dans des conditions normales de stabilite. Mais, si l'on est près d'un point critique, $\frac{\partial p}{\partial p}=0$, er les corrélations s'étendent a grande distance. Cela est en accord avec l'attente d'une clusterisation importante près du point critique.

On a done beaucoup de peine de créer une théorie pratique de la clusterisation pendant l'expansion. On s'est done rabattu sur des approximations plutot drastiques, à savoir le modele de coalescence sur lequel nous reviendrons plus loin, le modèle d'équilibre chimique, le modele des équations aux taux de réaction et enfin, récemment, une méthode du champ moyen agissant sur des corrélations de départ.

Nous nous arrêterons ici sur les équations aux réactions chimiques. Supposons que nous ayons deux espèces a et b. Les équations chimiques s'obtiennent en intégrant deux équations de LV couplées sur l'espace et les impulsions. On peut les réécire sous la forme

$$
\frac{d \rho_{i}}{d t}=-\frac{1}{y} \frac{d V}{d t} \rho_{i}-L_{i}+b_{i}
$$

où $L_{i}$ est un terme de perte et $G_{i}$ un terme de gain pour l'espece $i$. Supposons que I'on a la réaction $a+b \rightarrow c d$. Pour $i=a$, on aura done

$$
L_{a}=\int d^{3} p_{a} \int d^{3} p_{b} \sigma\left(v_{a b}\right) v_{a b} f_{a}\left(\vec{r}, \vec{p}_{a}, t\right) r_{b}\left(\vec{r}, \vec{p}_{b}, t\right)
$$

ou vab est la vitesse relative des particules. Comme $v_{a b}$ apparaft dans (5.8), on peut utiliser pour $f_{a}$ et $f_{b}$ leur représentation dans le référentiel où la matrice est au repos. Dans cette transformation $d^{3} p_{a} d^{3} p_{b}$ ne change pas. Dès lors, on peut ecrire pour $L_{i}$ et pour $G_{i}$

$$
\frac{d \rho_{a}}{d t}=-\frac{1}{V} \frac{d V}{d t} \rho_{a}-\rho_{a} \rho_{b}\langle\sigma v\rangle=
$$

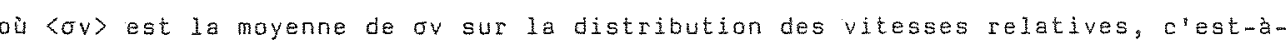
dire caractérisée par la température instantanée. A l'équilibre, on aura $\left(d \rho_{a} / d t=0\right)$

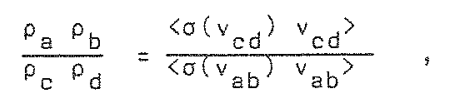

ou $\sigma\left(v_{c o}\right)$ est la section efficace qui détruit l'espece $c$. Le rapport (5.10) est une fonction de la température lacale (et aussi, peut-etre, de la densité). Sur la base de ce modele, il va de sai que la température extraite du rapport des concentrations sera différente et plus basse que la temperature extraite des spectres inclusifs. 


\title{
6.1. Introduction
}

En dépit du caractère transient de la réaction entre deux ions lourds, on peut tenter d'extraire l'équation d'état. L'idée est la suivante. On peut supposer sur la base de la figure 6.1 que l'état de compression maximale n'est pas trop

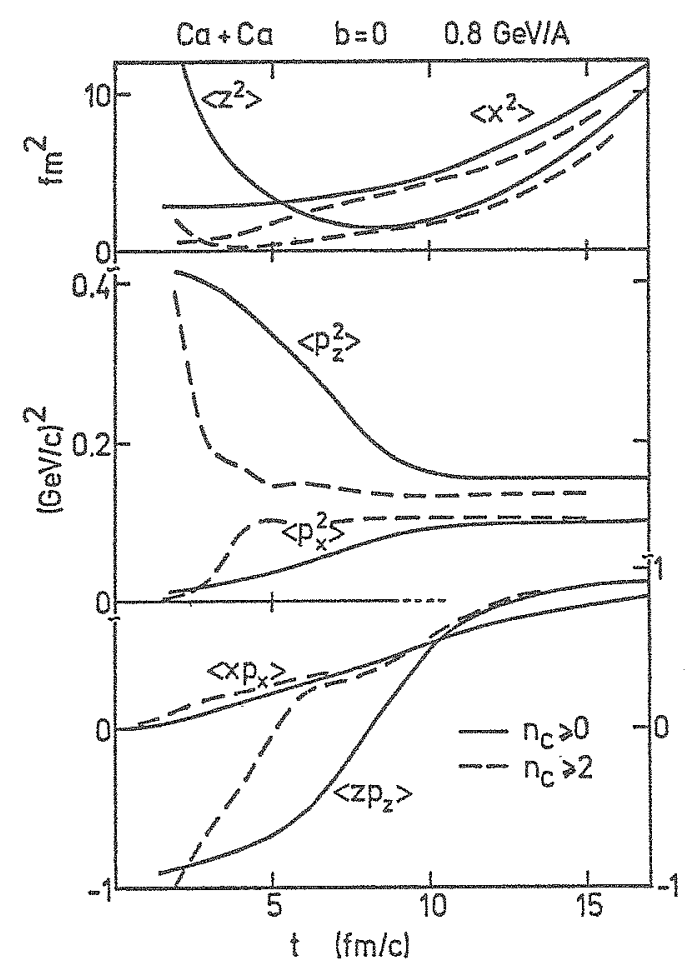

\author{
Figure 6.1
}

Evolution temporelle dans un modele de cascade de divers moments de la distribution à un corps $f_{1}(\vec{r}, \vec{p}, t)$. Les éléments non diagonaux $\left\langle x p_{x}\right\rangle$ et $\left\langle z p_{z}\right\rangle$ ont été normalisés en divisant par $\left(\left\langle x^{2}\right\rangle\left\langle p_{x}^{2}\right\rangle\right)^{\frac{1}{2}}$ ot $\left(\left\langle z^{2}\right\rangle\left\langle p_{z}{ }^{2}\right\rangle\right)^{\frac{1}{2}}$ respectivement. Les traits pleins se réfèrent à tous les nucléons tandis que les traits discontinus ne prennent en compte que les nucléons ayant fait deux collisions au moins. Adapté de la référence 29.

éloigné d'un état d'équilibre global (on s'aperçoit déjà que les resultats ne pourront etre que semi-quantitatifs). On étudie les prédictions pour les variables résistant à l'expansion d'une théorie qui décrit bien, croit-on, les effets hors équilibre. La C.I. semble la plus appropriée. Les différences entre observations et thérie doivent résulter, en partie au moins, des effets dinteraction. La manière de les extraire et de les quantifier n'est pas triviale et sera discutée plus ou moins en détail.

\subsection{Multiplicité de pions et énergie de compression}

La Figure 6.2 donne la comparaison théorie - expérience pour la multiplicité de pions. La C.I. prédit trop de pions. L'interpretation physique de cette discordance a été avancée par 5tock. Si l'on admet que l'abondance des pions et des $\Delta$ est fonction directe de l'energie cinétique disponible, on peut attribuer les prédictions de la C.I. comme étant dues à une trop grande quantité d'énergie 


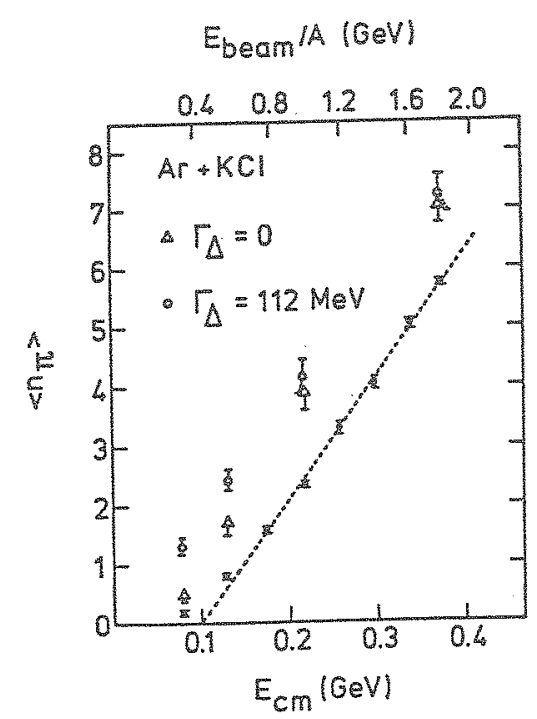

\section{Figure 6.2}

Multiplicité des pions négatifs dans les collisions centrales d'Ar $+K C l$. Les points sont les résultats expérimentaux. Ils sont comparés aux prédictions d'un modèle de cascade intranucléaire où la résonance $\Delta a$, soit son temps de vie normal (cercles), soit un temps de vie infini (triangles).

cinétique au moment de la compression maximale et donc à un manque d'énergie de campression.

On peut quantifier ceci. Pour un équilibre thermique et chimique dans un volume $Y$ à température $T$, l'énergie disponible $E$ dans le système peut s'écrire

$E=\frac{4}{2 \pi^{2}} N_{N}\left(m_{N}+\frac{3}{2} T\right)+\frac{16}{2 \pi^{2}} N_{\Delta}\left(m_{\Delta}+\frac{3}{2} T\right)+\frac{3}{2 \pi^{2}} m_{\pi}{ }^{2} T{ }^{2}\left\{3 K_{2}\left(\frac{m_{\pi}}{T}\right)+\frac{m_{\pi}}{T} K_{1}\left(\frac{m_{\pi}}{T}\right)\right\}$

et bien sûr

$$
\begin{aligned}
& \frac{N_{\Delta}}{N_{N}}=4\left(\frac{m_{\Delta}}{m_{N}}\right)^{3 / 2} e^{-\frac{m_{\Delta}-m_{N}}{T}} \\
& N_{\pi}=\frac{3 V}{2 \pi^{2}} m_{\pi}^{2} T k_{2}\left(\frac{m_{\pi}}{T}\right),
\end{aligned}
$$

où l'on a fait $k=m=c=1$, et où $k_{2}$ est la fonction de Bessel modifiée habituelle. Le nombre de baryons est fixé par

$$
N_{N}+N_{\Delta}=A
$$

On peut déterminer $N_{N}, N_{\Delta}, N_{\pi}$ et $T$ si $A, E$ et $V$ sont connus. On peut en éliminant $N_{N}, N_{B}, T$ (ce qui n'est pas nécessairement facile) écrire $(p=A / V)$

$$
N_{\pi}=f_{E C}(E / A, D) \text {. }
$$




$$
N_{\pi}=r_{E C}\left(U / A-E_{C}(0), 0\right) .
$$

Ici, on a fait l'hypothèse implicite que les propriétés d'équilibre entre les espé cesne sont pas modifiéespar la compression. En d'autres termes, on suppose qu'il n'y a pas.d'effets du milieu.

On peut penser que dans la réalité l'équilibre chimique n'est pas réalisé, à cause deffets dynamiques. Si ceux-ci sont pris en compte par la cascade intranucléaire, on pourre néanmoins écrire

$$
N_{\pi}=f_{C I}\left(U / A-E_{C}(\rho), \rho\right),
$$

ou $\hat{f}_{C I}$ est une fonction inconnue, mais qui est décrite numériquement par la C.I. Bien sar, comme il n'y a pas d'interaction dans la C.I., on peut représenter les prédictions de celle-ci pas

$$
N_{\pi}^{C I}=F_{C I}(U / A, D),
$$

où U est l'énergie disponible dans le c.m. du système, ce qui est aussi une approximation. Dès lors, on tire $E_{c}(\rho)$ par comparaison entre thérie et expérience. En adoptent cette procédure, an n'évite pas un certain arbitraire. En ef fet, on doit tirer $\rho$ de la cascade, et rien ne dit que la prédiction de la C.I. ne devrait pas etre affectée par la présence d'énergie de compression. Il existe une controverse sur ce qu'il faut retirer dans (6.7) pour tenir compte du manque d'énergie de liaison dans la cascade, mais de toute manière, il en résulte (voir Figure 6.3) une fonction $E_{c}(\rho)$ qui est du genre "équation d'état" dure. Les points ouverts indiquent les résultats lorsque l'on utilise $(6.6)$ et que l'on détermine $\rho$ par un calcul de RH.

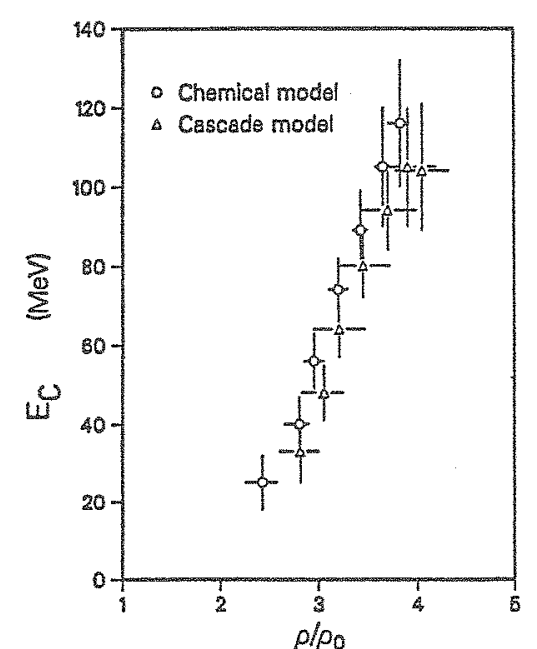

\section{Figure 6.3}

Energie de compression extraite de la comparaison entre l'expérience et la prédiction de la C.I. (triangle) et celles diun modèle d'équilibre chimique (points). Voir texte, Les donnés se réfèrent essentiellement au système Ar + KCl. Adapté de la référence 30 . 
Il n'y a guère de doute qu'il manque de l'énergie de compression dans la C.I. ; neanmoins il sied de rappeler ici que le résultat de la figure 6.3 est obtenu au prix de trois hypothèses majeures:

(1) le taux de pions est donné par un processus, réactif paurait-on dire, de cascade intranucléaire;

(2) le taux de pions dépend de la quentite $E_{t h}(0, T)$, ce qui peut perastre un peu étonnant, car à l'équilibre, le taux de pions ne devrait dépendre que des grandeurs decrivant l'équilibre;

(3) les propriétés a une particule ne sont pas changées. Ceci peut parastre suspect en ce qui concerne le pion.

Il faut remerquer que, en quelque sorte, et moyennant une hypothèse supplementaire sur la teneur de la fonction $E_{t h}$, la multiplicite de pions sert de thermomètre de la matière chaude. On peut d'ailleurs utiliser (6.2) pour déterminer la température de la boule de feu, puisqu'a ce moment, les pions finaux existent surtout sous la forme de résonances $\Delta$.

\subsection{Formation de composites et entropie}

Siemens et Kapusta ${ }^{31}$ ont été les premiers à suggérer que la multiplicité des composites pourrait donner une mesure de l'entropie. Ainsi que nous l'avons dit, la quantité cruciale ici est la fonction de distribution à deux (ou trois,...) la quantité cruciale ici est la fonction dion s'intéresse aux deutérons, par exemple, on peut écrire 5,3

$N_{\text {"du }}=\frac{3}{4} \int d^{3} R \int d^{3} p \int d^{3} r d^{3} p f_{2}^{n p}\left(\vec{R}+\frac{\vec{r}}{2}, \vec{k}-\frac{\vec{r}}{2}, \frac{\vec{p}}{2}+\vec{p}, \frac{\vec{p}}{2}-\vec{p}\right) g_{d}(\vec{r}, \vec{p})$

ou go est la représentation de wigner de la fonction d'onde du deutéron. Le facteur $3 / 4$ tient compte des spins. L'équation (6.9) compte tout simplement toutes les corrélations du Eype deutéron qui existent dans le systeme à la fin de la collision. Encore une fois, ces corrélations peuvent exister dans un "cluster" plus grand, d'ou les guillemets introduits dans (6.9). Pour des "clusters" jusque la particule alpha ${ }^{5}$

$$
N_{n d u}=N_{d}+\frac{3}{2}\left(N_{t}+N_{3 e}\right)+3 N_{\alpha}
$$

Si lon suppose, comme dans le modèle de coalescence, que les deutérans apparaissent au hasard, c'est-à-diresi $f_{2}^{n p} \approx f_{1}^{n} f_{1}^{p}$, on peut écrire, en admettant que $9_{d}$ est une fonction très piquée

$$
R_{d p}=\frac{N_{n d "}}{N_{\text {"p" }}}=6 \frac{N}{A}\left\langle F_{1}\right\rangle
$$

où est la multiplicité de charge

$$
N p^{n}=\int d^{3} r d^{3} p f_{1}^{p}(\vec{r}, \vec{p}),
$$

ou 〈f $\rangle$ represente la moyenne de la distribution $f_{1}$ sur elle-méme. Dans (6.11) nous avons supposé que $f_{1}^{n} \approx f_{1}$ et $N$ et $A$ représentent le nombre de neutrons 


$$
\begin{aligned}
& f_{1}(\vec{r}, \vec{p})=\frac{A}{\left(\pi R_{p} \sqrt{2 m T}\right)^{3}} \exp \left[-\frac{r^{2}}{R_{p}}-\frac{p^{2}}{2 m T}\right], \\
& g_{d}(\vec{r}, \vec{p})=\frac{1}{\left(\pi r_{0} p_{0}\right)^{3}} \exp \left[-\frac{r^{2}}{r_{0}^{2}}-\frac{p^{2}}{p_{0}^{2}}\right],
\end{aligned}
$$

où $\quad F_{0} p_{0} \approx H$, ainsi que le demande la quantification. Alors, (6.11) devient

$$
\frac{N{ } d "}{N p^{\prime}}=6 \frac{N}{A}\left\langle f_{1}\right\rangle \frac{1}{\left(1+\frac{r_{0}^{2}}{2 R_{p}^{2}}\right)^{3 / 2}} \frac{1}{\left(1+\frac{p_{0}^{2}}{m T}\right)^{3 / 2}} .
$$

D'un autre coté, I'entropie qui, pour un gaz classique, vaut (généralisation de I'équation $(2.43))^{33}$

$$
\frac{S}{A}=1-\left\langle\ln F_{1}\right\rangle
$$

s'écrit, dans les mêmes conditions que ci-dessus

$$
\frac{S}{A}=1-\left\langle\ln f_{1}\right\rangle+\ln 2-\frac{N}{A} \ln \frac{N}{A}-\frac{Z}{A} \ln \frac{Z}{A} .
$$

si $f_{1}$ est proche de $(6.13)$, on peut écríre

$$
\frac{5}{A}=-3(1-\ln 2)+\ln \left\langle f_{1}\right\rangle .
$$

Finalement, en comparant $(6.15)$ et $(6.18)$, on a

$\frac{5}{A}=4+\ln \frac{3}{2}+\frac{Z}{A} \ln \frac{N}{Z}-\frac{3}{2} \ln \left(1+\frac{r_{0}^{2}}{2 R_{p}^{2}}\right)-\frac{3}{2} \ln \left(1+\frac{P_{0}^{2}}{m T}\right)-\ln R_{d p}$.

Pour $N=2$ et en négligeant les effets de taille finie du deutéron, on retrouve pour un système uniforme, la fameuse formule 31

$$
S / A=3.95-\text { en } R_{d p} \text {. }
$$

En général, $p_{0}^{2}$ est nettement plus petit que $m T$, ce qui fait que la correction de taille est 1 a plus importante. Si maintenant, on regarde le rapport $R_{\text {dp }}$ (équation (6.75)) pour un système donné et pour des multiplicites de plus en plus grandes, on s'attend à ce que $R_{d p}$ croisse, car $R_{p}$, la taille de la zone d'interaction, augmente avec la multiplicité. C'est ce que l'on observe expérimentalement (voir figure 6.4) sur les systemes $\mathrm{Nb}+\mathrm{Nb}$ et $\mathrm{Ca}+\mathrm{Ca}$ qui ant été étudiés systématiquement ${ }^{34}$. La dépendance de $R_{\text {dp }}$ avec la multiplicite ou, ce qui revient au meme, avec le paramètre d'impact, est prévue également par la $C . I$. (Fiqure 6.5) qui montre aussi que la formule $(6.20)$ n'est valable que pour les 

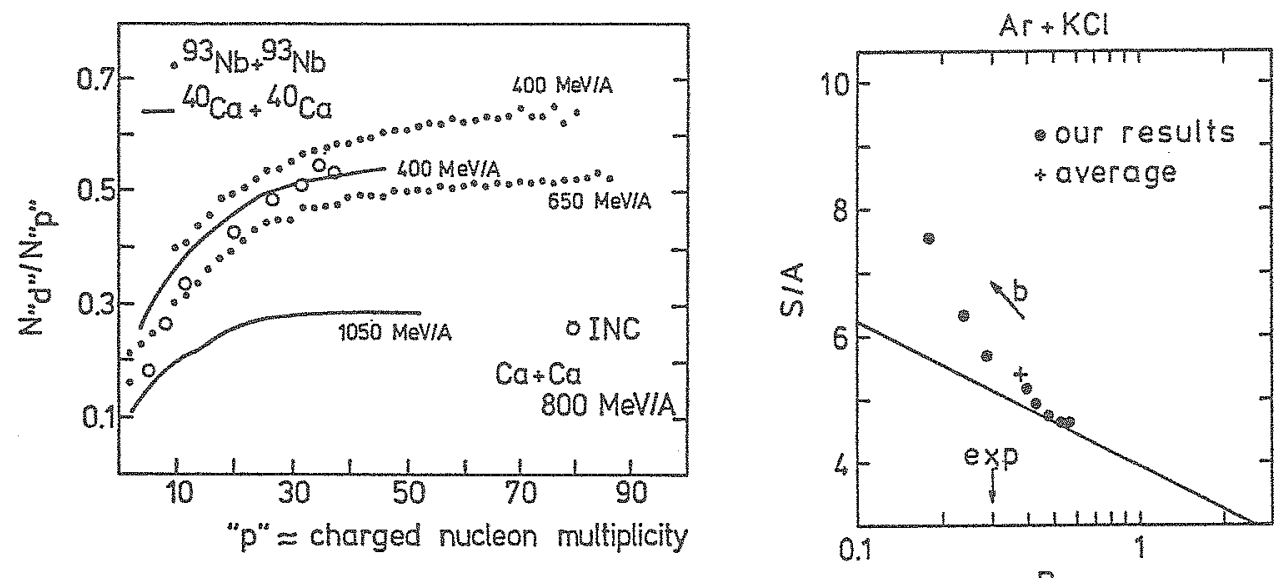

$R_{d p}$

Figure 6.4. Valeurs expérimentales du rapport "d"/ "p" en fonction de la multiplicite de charge. Les cercles blancs sont les prédictions théariques d'un modèle de C.I. ${ }^{35}$ pour le système $\mathrm{Ca}+\mathrm{Ca}$ \& $800 \mathrm{MeV} / \mathrm{A}$.

rigure 6.5. Calcul (points) par un modèle de C.I. du rapport $R_{d p}$ (par la formule $(6.9)$ ) et de l'entropie (équation (6.17)) pour divers parametres d'impact allant de façon régulière de 0 à $7.15 \mathrm{fm}$ selon le sens de la flèche. La droite en traits pleins représente l'équation (6.20)).

grandes multiplicités. Il est donc indiqué de faire des comparaisons pour les grandes multiplicités seulement et non comme dans le passé sur les sections efficaces inclusives. Aussi seules sont valables probablement les valeurs de l'entropie extraites des grandes multiplicités. C'est ce qui est fait dans la figure 6.6. Elle montre qu'une entropie de 4 à 5 unités par nucléon est produite, à peu près

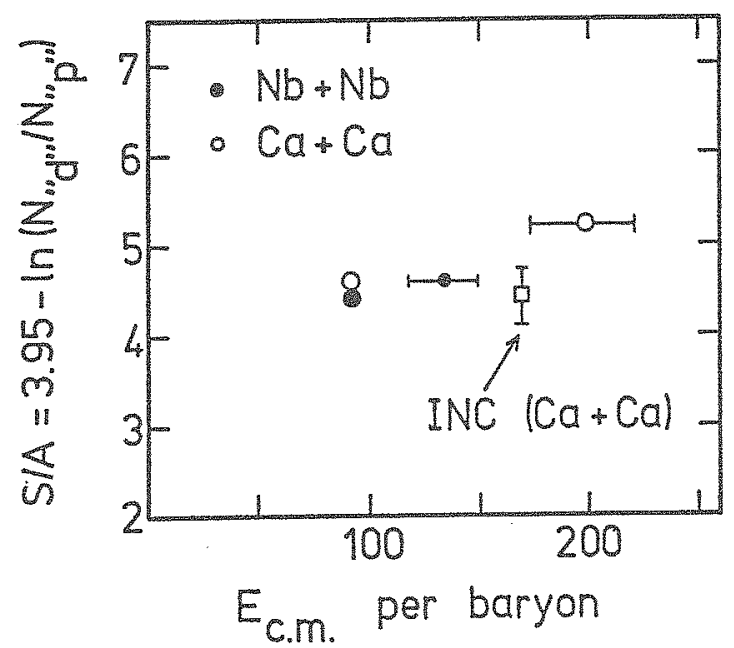

Figure 6.6

Comparaison entre les valeurs expérimentales de $R_{\text {do }}$ (ou de l'entropie selon (6.19)) tirées des grandes multiplicités et les prédictions de la rérérence 35. 
une demi-unité de plus que la prédiction de la C.I. Ceci correspondrait à une equation d'état "molle". Ce résultat est très dérangeant en regard avec ce que l'on a discute sur la production de pions.

Cependant, il faut noter que dans la référence 36 , les résultats sont analysés grâce à un modèle d'équilibre chimique, mais qui tient compte de la désintégration des espèces instables. Cette dernière proprieté est importante, car elle provoque une reduction des taux des particules lourdes (ici, plus lourd que la particule alpha). Grâce a ce modèle, une entropie de l'ordre de deux unités est extraite des résultats expérimentaux, ce qui à nouveau donnerait une-équation d'état dure $\left(\sim E_{C} \sim 120\right.$ MeV pour $\left.\rho / p_{0} \approx 3\right)$.

Une autre considération, très séduisante, a été faite par Kapusta et al. 37 . qui considèrent que le système durant l'expansion peut entrer dans la phase de coexistence gaz-liquide et se scinde en une partie liquide de basse entropie et une partie gazeuse de grande entropie. Ils assimilent la partie liquide associée aux gros fragments et la partie gazeuse aux petits fragments. On peut ainsi expliquer les résultats expérimentaux avec une entropie de départ de l'ordre de 2 unités, mais en utilisant une densité de freeze-out de $0.1 \rho_{0}$, ce qui sembie vraiment très bas 38 .

En résumé, la situation est assez confuse pour l'instant, bien qu'il ne fait nul doute que la production de fragments est reliée à l'entropie, car celle-ci mesure en quelque sorte le degré de désordre de la matière.

\subsection{Ecoulement coliectif}

Cette question, toujours très débattue actuellement, est vaste et complexe. Nous ne pouvons en donner ici qu'un raccourci. L'écoulement collectif se réfère a la déviation de la matiére dans une direction privilégié, comme on peut le concevoir à l'examen de la figure 3.3. Cet écoulement résulte, comme le sugqère la Figure 3.5, de la pression accumulée dans la région centrale qui pousse sur les parties extérieures du systeme. Pour toute une série de raisons qui ont trait à la moyenne sur les paramètres d'impact et aux fluctuations (non présentes dans un calcul hydrodynamique), l'écoulement collectif ne peut être décelé aisément dans les sections efficaces inclusives. L'analyse se fait grace aux variables globales, en général grace au tenseur de sphéricité

$$
Q_{i j}=\sum_{\nu} \frac{1}{2 m_{v}} p_{i}^{\nu} p_{j}^{\nu},
$$

où $\vec{p}^{\nu}$ est l'impulsion (dans le com.) du $\nu^{i e ̀ m e}$ éjectile. Ce tenseur peut etre représenté par ses valeurs propres $\lambda_{1} \geq \lambda_{2} \geq \lambda_{3}$ et trois angles. En général cependant, on se contente de l'aspect

$$
a_{1}=\lambda_{1} / \lambda_{3}
$$

et de l'angle $\theta$ entre le premier vecteur propre $\vec{e}_{\text {, }}$ et l'axe du faisceau. Ces quantités suffisent en genéral pour caractériser les événements ordinaires. Ainsi un événement de thermalisation complète correspond à $q_{1} \approx 1$. De meme, un écoulement du type hydrodynamique correspond $a q_{1}>1$ et un angle $\theta$ bien 
On regarde généralement la distribution des événements par unité de $\cos \theta$ (dN/ (don/d cos née dans la figure 6.7. On peut voir que la C.I. indique pour $\mathrm{Nb}+\mathrm{Nb}$ une dévi-

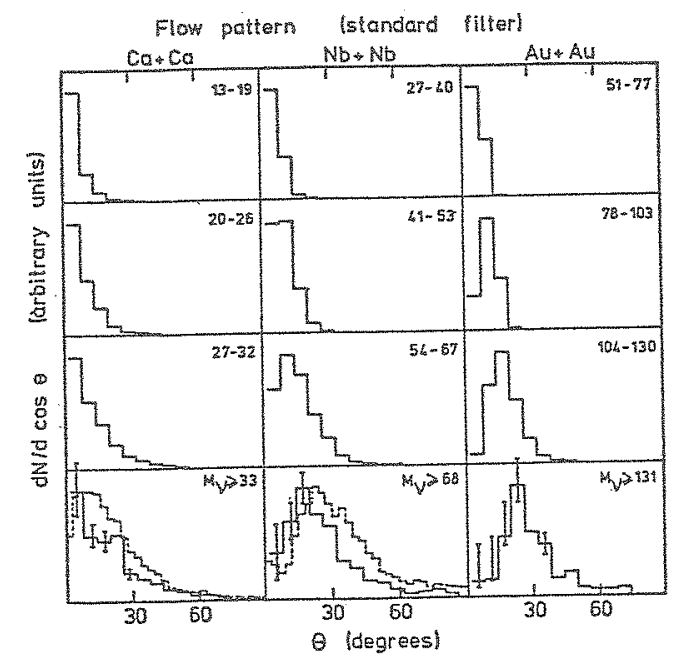

\section{Figure 6.7}

Calcul de la distribution de l'angle d'écoulement par un modèle de 20 (traits pleins). Les pointillés donnent les résultats expérimentaux pour les plus grandes multiplicités de particules chargés 39 . M est le nombre (calculé) de nucléons participants.

ation trop petite vis-à-vis de l'expérience. En première analyse, on peut dire qu'il manque de la pression dans la C.I. et que l'expérience demande une équation d'état "dure". Une analyse faite par stöcker et al.s à l'aide d'une simulation complete de l'equation de $L V$, indique une valeur de $E_{c}$ de 80 MeV pour une densité de 3040 . Une analyse du type de celle effectuée pour la multiplicité de pions donne 80 Mev pour une densité de $2.75 \rho_{0}{ }^{41}$.

\subsection{Synthèse}

Avec les trois données expérimentales ci-dessus, nous pouvons estimer la température, I'entropie et la pression de la matière dense. Il faut cependant garder en kête que ces résultats ne sont que semi-quantitatifs et ne peuvent etre pris qu'à un facteur 2 prés, dans l'état actuel des analyses. Dans la figure 6.8

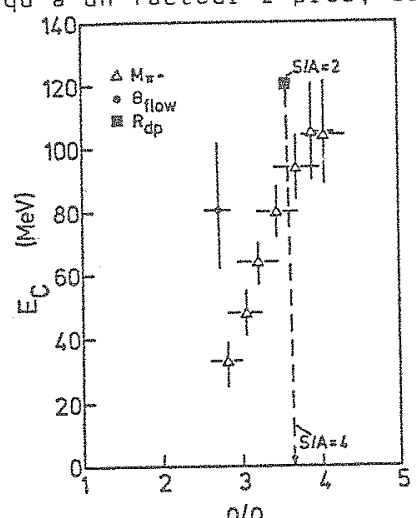

\section{Figure 6.8}

Résumé des indications trouvées sur $E_{c}$ en utilisant le principe énoncé dans la section 6.1 
nous tentons de donner un résumé de ce qui est obtenu en gardant le principe énoncé au début de cette section. Même en tenant compte d'un facteur 2 et en se souvenant que la question de l'entropie reste entiere, on peut avancer que l'on a une forte indication, sinon une évidence, pour une equation d'état "dure".

\section{CONCLUSION}

Nous avons analysé la dynamique des collisions entre ions lourds relativistes dans la perspective de la détermination des propriétés de la matière dense formée transitoirement. L'état actuel de la question fait place a un certain optimisme quant à la possibilité d'une telle détermination et vient récompenser l'effort considérable produit pendant les dix dernières années. Nous en rappelons rapidement les grandes etapes : (1) mise en evidence de collisions violentes avec disposition d'une grande énergie d'excitation; (2) élaboration d'outils capables d'étudier avec une certaine confiance les effets dynamiques de non-équilibre; (3) la sélection d'observables résistant à l'expansion ; (4) l'extraction par l'étude de ces observables de renseignements sur l'équation d'état.

Ces renseignements sont obtenus au prix d'hypothèses raisonnables certes, mais dont la justification n'est pas totale. Rappelons que l'une d'entre elles fait jouer un grand róle à l'énergie de compression à $T=0$, même lorsque le système atteint des températures très élevés. Ainsi que nous l'avons vu, ce travail d'analyse donne de fortes indications pour une equation d'état "dure". Du point de vue quantitatif, cependant, on n'est pas encore très avancé. Les divers żavaux récents permettant de résoudre numériquement l'equation de Landau-Vlassov sont très prometteurs.

A plus basse énergie, les conditions de non-équilibre sant vraisemblablement assez proches de ce qu'elles sont à haute énergie. Cependant, les observables ne sont peut-être pas comparables car, à basse énergie, la production de composites est particulièrement abondante. Le problème le plus important dans cette région n'est peut-etre pas l'étude de I'équation d'état, mais la compréhension du processus de fragmentation. A ce propos, l'extension des modeles développés à haute énergie devrait être réalisée. L'entropie devrait y jouer également un grand role.

\section{REFERENCES}

1. C.F. Chapline, H.H. Johnson, E. Teller and M.S. Weiss, Phys.Rev. D8 (1973) 4302 .

2. Y. Yariv and Z. Fraenkel, Phys.Rev. C20 (1979) 2227.

3. J. Cugnon, T. Mizutani and J. Vandermeulen, Nucl.Phys. A352 (1981) 505.

4. M. Sano et a1., Phys.lett. B156 (1985) 27.

5. G. Bertsch and 3. Cugnon, Phys.Rev. C24 (1981) 2514.

6. R. Stock et al., Phys.Rev.Lett. 44 (1980) 1243.

7. B. Friedman and V.R. Pandharipande, Nucl, Phys. A361 (1981) 502.

8. 3. Cugnon and $A$. Lejeune, à paraitre.

9. B.D. Serot and J.D. Walecka, à paragtre dans "Advances in Nuclear Physics". 
10. P. Bonche, cette école.

11. R. Babinet, cette école.

12. 5. Nagamiya, preprint 1984 .

13. G. Bertsch, in "Progress in Particle and Nuclear Physics", vol. 4, D. Wilkinson, ed., Pergamon, Oxford (1980), p. 483.

14. A.A. Amsden, G.F. Bertsch, F.H. Harlow and J.R. Nix, Phys.Rev.lett. 35 (1975) 905.

15. H. Stäcker, J.A. Marhun and W. Greiner, Phys.Rev.Lett. 44 (1980) 725.

16. G. Buchwald, L.P. Csernai, J.A. Maruhn and W. Greiner, Phys.Rev. C24 (1981) 135.

17. A. Sandoval et al., Phys.Rev. C21 (1980) 1321.

18. J.R. Nix and D. Strottman, Phys.Rev. C23 (1981) 2548.

19. J. Cugnon, Nucl.Phys. A387 (3982) 1910.

20. J. Cugnan and D. L'Hôte, à paraitre.

21. L.P. Kadanoff and G. Baym, "Quantum Statistical Mechanics, Benjamin, New York (1962).

22. S.E. Koonin, thesis.

23. J. Knoll and J. Randrup, Nucl. Phys. A324 (1979) 445.

24. J. Knoll, Phys.Rev. C20 (1979) 773.

25. J.P. Bondorf, S.I.A. Garpman and J. Zimanyi, Nucl.Phys. A296 (1978) 320.

26. J. Cugnon, Phys.Lett. 1358 (1984) 374.

27. P.J. Siemens and J.0. Rasmussen, Phys.Rev.Lett. 42 (1979) 844.

28. H. Stöcker, GSI Meeting, 0ct. 1984, pubI. GSI-85-10.

29. 3. Cugnon and J. Vandermeulen, "Winter College on Nuclear Physics", Trieste, March 1984, a publier par W.S.P.C..

30. J.W. Harris et al., Phys.Lett. 153B (1985) 377.

31. P.J. Siemens and J.I. Kapusta, Phys.Rev.Lett. 43 (1979) 1486.

32. M. Gyulassy, K. Frankel and E.A. Remler, Nucl. Phys. A402 (1983) 596.

33. C. Kittel, "Eléments de Physique Statistique", Dunod (1961).

34. K.G.R. Doss et al., preprint GSI-85-4.

35. G. Bertsch and J. Cugnon, non publíe.

36. B.V. Jacak, H. Stöcker and G.D. Westfall, Phys.Rev. C29 (1984) 1744.

37. A.L. Goodman, 3.I. Kapusta and A.2. Mekzian, Phys.Rev. C19 (1984) 851.

38. L.P. Csernai, Phys.Rev.Lett. 54 (1985) 639

39. H. - A. Gustaffsont? Phys.Rev.Lett. 52 (1984) 1590.

40. J.J. Molitoris, J.B. Hoffer, H. Kruse and H. Stäcker, Phys.Rev.Lett. 53 (1984) 899.

41. J. Cugnon, nan publié. 
